\title{
SiPM-matrix readout of two-phase argon detectors using electroluminescence in the visible and near infrared range
}

\author{
The DarkSide-20k collaboration ${ }^{\star}$
}

Received: 6 April 2020 / Accepted: 23 December 2020 / Published online: 15 February 2021

(C) The Author(s) 2021

\begin{abstract}
Proportional electroluminescence (EL) in noble gases is used in two-phase detectors for dark matter searches to record (in the gas phase) the ionization signal induced by particle scattering in the liquid phase. The "standard" EL mechanism is considered to be due to noble gas excimer emission in the vacuum ultraviolet (VUV). In addition, there are two alternative mechanisms, producing light in the visible and near infrared (NIR) ranges. The first is due to bremsstrahlung of electrons scattered on neutral atoms ("neutral bremsstrahlung", NBrS). The second, responsible for electron avalanche scintillation in the NIR at higher electric fields, is due to transitions between excited atomic states. In this work, we have for the first time demonstrated two alternative techniques of the optical readout of two-phase argon detectors, in the visible and NIR range, using a silicon photomultiplier matrix and electroluminescence due to either neutral bremsstrahlung or avalanche scintillation. The amplitude yield and position resolution were measured for these readout techniques, which allowed to assess the detection threshold for electron and nuclear recoils in two-phase argon detectors for dark matter searches. To the best of our knowledge, this is the first practical application of the $\mathrm{NBrS}$ effect in detection science.
\end{abstract}

\section{Introduction}

In two-phase noble liquid detectors for dark matter searches and low-energy neutrino experiments, the scattered particle produces two types of signals [1]: that of primary scintillation, recorded promptly ("S1") and that of primary ionization, which is recorded with a delay ("S2"). The S1 and S2 signals are typically recorded by photomultiplier tubes (PMTs) [2] or silicon photomultiplier (SiPM) matrices [3], adapted for operation at cryogenic temperatures.

The detection of S1 serves to find the $\mathrm{z}$ coordinate of the interaction vertex (by the S1-S2 time difference). In addition, it is very useful for effective selection between nuclear

\footnotetext{
*e-mails: ds-ed@lngs.infn.it (corresponding author)
}

and electron recoils, using either the $\mathrm{S} 1$ pulse-shape or the $\mathrm{S} 1 / \mathrm{S} 2$ ratio for discrimination. However, the $\mathrm{S} 1$ detection is not a must: in particular, at lower recoil energies where the $\mathrm{S} 1$ signal is too weak, experiments can resort to an S2-only operation mode $[4,5]$. Accordingly, in this paper we confine ourselves to the issue of S2-only signal detection, leaving issues related to $\mathrm{S} 1$ to future studies.

S2 detection in dual-phase detectors relies on proportional electroluminescence (EL) in the noble gas [6,7]. In argon, the ordinary ("standard") mechanism of proportional electroluminescence is considered to be due to vacuum ultraviolet (VUV) emission (around $128 \mathrm{~nm}$ ) of noble gas excimers $\operatorname{Ar}_{2}^{*}\left({ }^{1,3} \Sigma_{u}^{+}\right)$produced in three-body atomic collisions of excited atoms $\mathrm{Ar}^{*}\left(3 \mathrm{p}^{5} 4 \mathrm{~s}^{1}\right)$, which in turn are produced by drifting electrons in electron-atom collisions: see review [7].

While present two-phase argon detectors rely on PMTs, the baseline design option for the future dark matter detector DarkSide-20k [3] employs SiPM matrices to record ordinary electroluminescence in the VUV. The sensitivity of PMTs and SiPMs is limited to the visible or NUV range $[8,9]$. The development of SiPMs with VUV sensitivity, namely at $128 \mathrm{~nm}$, is still at the R\&D stage [10], since such SiPMs are windowless [11] which limits their use in cryogenic conditions and over large sensitive areas. It is thus necessary to convert the VUV into visible light using a wavelenght shifter (WLS), namely tetraphenyl-butadiene (TPB). An issue with TPB is that it may not be stable over long time scales, in particular due to its dissolving in liquid $\operatorname{Ar}$ [12] and peeling off from the substrate under cryogenic conditions [13]. Another known issue is related to difficulties in achieving uniform levels of WLS deposits over large detector areas.

Alternative readout techniques for two-phase argon detector, proposed elsewhere $[14,15]$ and based on "non-standard" electroluminescence in the visible and near infrared (NIR) range, could allow detector operation without WLS. In this work, we demonstrate the successful S2-only performance of a two-phase argon detector with SiPM-matrix optical readout, in the visible and NIR range, using two such alternative readout techniques. 
This study was performed using the experimental setup of the Novosibirsk group of the DarkSide collaboration.

\section{Alternative concepts of SiPM-matrix readout of two-phase argon detectors}

In argon, ordinary electroluminescence (in the VUV, around $128 \mathrm{~nm}$, see Fig. 1) goes via $\mathrm{Ar}^{*}\left(3 \mathrm{p}^{5} 4 \mathrm{~s}^{1}\right)$ atomic excited states [7] and thus has a threshold for the reduced electric field of about $4 \mathrm{Td}$ [15], which is defined by the energy threshold for Ar atom excitation. The reduced electric field is defined as $\mathcal{E} / N$ expressed in $\mathrm{Td}$ units $\left(1 \mathrm{Td}=10^{-17} \mathrm{~V} \mathrm{~cm}{ }^{2}\right)$ corresponding to $0.87 \mathrm{kV} / \mathrm{cm}$ in gaseous argon at a pressure of $1.00 \mathrm{~atm}$ and a temperature of $87.3 \mathrm{~K}$, where $\mathcal{E}$ is the electric field and $N$ is the atomic density.

In addition to the ordinary EL mechanism, a concurrent EL mechanism, based on bremsstrahlung of drifting electrons scattered on neutral atoms (so-called "neutral bremsstrahlung", $\mathrm{NBrS}$ ), has been recently revealed $[15,16]$. It was shown that the $\mathrm{NBrS}$ effect can explain two remarkable properties of proportional electroluminescence: photon emission below the Ar excitation threshold and the substantial contribution of a non-VUV spectral component. $\mathrm{NBrS}$ electroluminescence has a continuous emission spectrum, extending from the UV to the visible and NIR range: see Fig. 1.

At higher electric fields (above $8 \mathrm{Td}$ ), another "nonstandard" EL mechanism comes into force, namely that of electroluminescence in the NIR due to transitions between excited atomic states [7,14,17-20]: $\operatorname{Ar}^{*}\left(3 p^{5} 4 p^{1}\right) \longrightarrow$ $\operatorname{Ar}^{*}\left(3 p^{5} 4 s^{1}\right)$. It has a line emission spectrum in the range of 700-850 nm (Fig. 1). Similarly to the ordinary mechanism, the excited $\mathrm{Ar}^{*}\left(3 \mathrm{p}^{5} 4 \mathrm{p}^{1}\right)$ atoms are produced by drifting electrons in electron-atom collisions. This mechanism is particularly noticeable at even higher fields, above $30 \mathrm{Td}$, where avalanche multiplication takes place, accompanied by secondary scintillation("avalanche scintillation") $[18,21]$.

Figure 2 presents all known experimental data on reduced EL yield in argon for all known EL mechanisms: for NBrS electroluminescence at wavelengths below $1000 \mathrm{~nm}$, for ordinary electroluminescence in the VUV and for electroluminescence in the NIR. In addition, Fig. 1 shows their photon emission spectra, along with the spectral response of the SiPMs used in the present study.

The "standard" concept of SiPM matrix readout of twophase argon detectors is depicted in Fig. 3. In this concept the SiPM matrix is coupled to the EL gap via a wavelength shifter (WLS).

Figure 4 illustrates two alternative readout concepts proposed elsewhere $[14,15,19]$ and realized in the present study. These are based on $\mathrm{NBrS}$ electroluminescence and avalanche scintillations in the NIR, respectively.

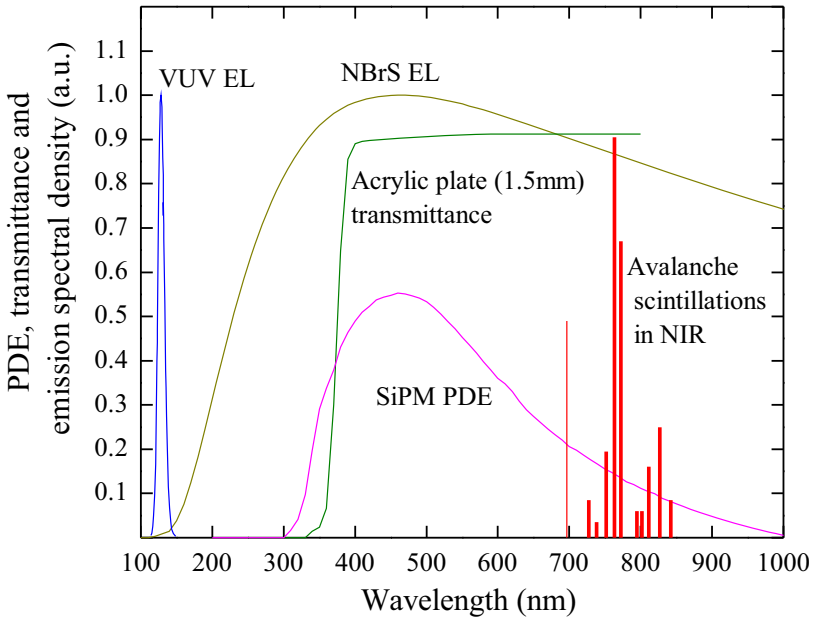

Fig. 1 Photon emission spectra in gaseous Ar due to ordinary scintillations in the VUV measured in [22], NBrS electroluminescence at $8.3 \mathrm{Td}$ theoretically calculated in [15] and avalanche scintillations in the NIR measured in $[17,18]$. Also shown are the photon detection efficiency (PDE) of the SiPMs (MPPC 13360-6050PE [23]) at an overvoltage of $5.6 \mathrm{~V}$ obtained from [24] and the transmittance of the acrylic plate (1.5 mm thick) in front of the SiPM matrix, used in this study

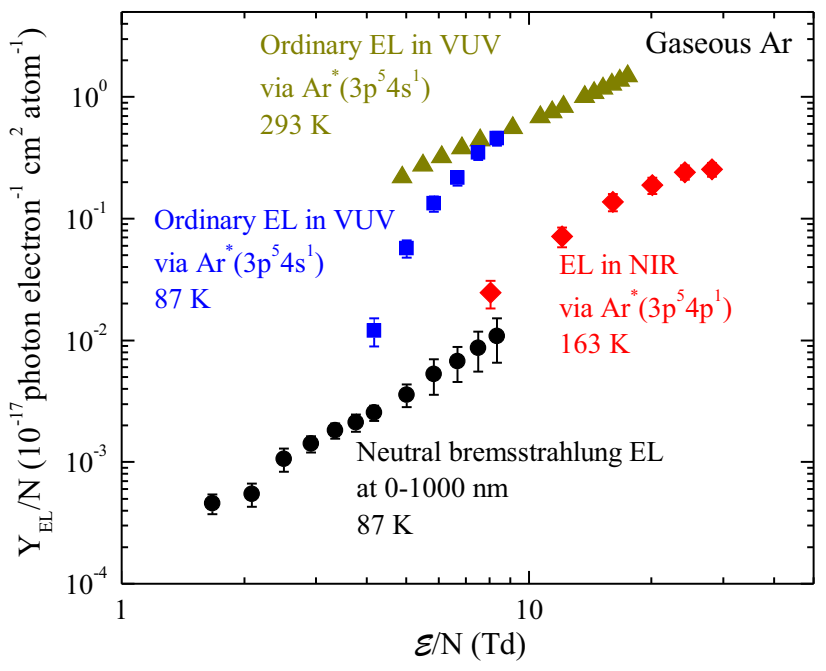

Fig. 2 Summary of experimental data on the reduced EL yield in argon for all known electroluminescence (EL) mechanisms: for NBrS EL at wavelengths of $0-1000 \mathrm{~nm}$, measured in [16] at $87 \mathrm{~K}$; for ordinary EL in the VUV, going via $\mathrm{Ar}^{*}\left(3 \mathrm{p}^{5} 4 \mathrm{~s}^{1}\right)$, measured in [16] at $87 \mathrm{~K}$ and in [25] at $293 \mathrm{~K}$; for EL in the NIR going via $\operatorname{Ar}^{*}\left(3 p^{5} 4 p^{1}\right)$, measured in [14] at $163 \mathrm{~K}$

In the first alternative concept [15], the EL gap is read out directly in the visible and NIR range, using a SiPM matrix directly coupled to the EL gap. In the second alternative concept $[14,19]$, the EL gap is read out indirectly, using a combined THGEM/SiPM-matrix multiplier coupled to the EL gap, the THGEM being operated in electron avalanche mode. The advantage of these concepts is operating without a WLS. As noted above, this may lead to more stable operation of two-phase argon detectors, avoiding the problems of 


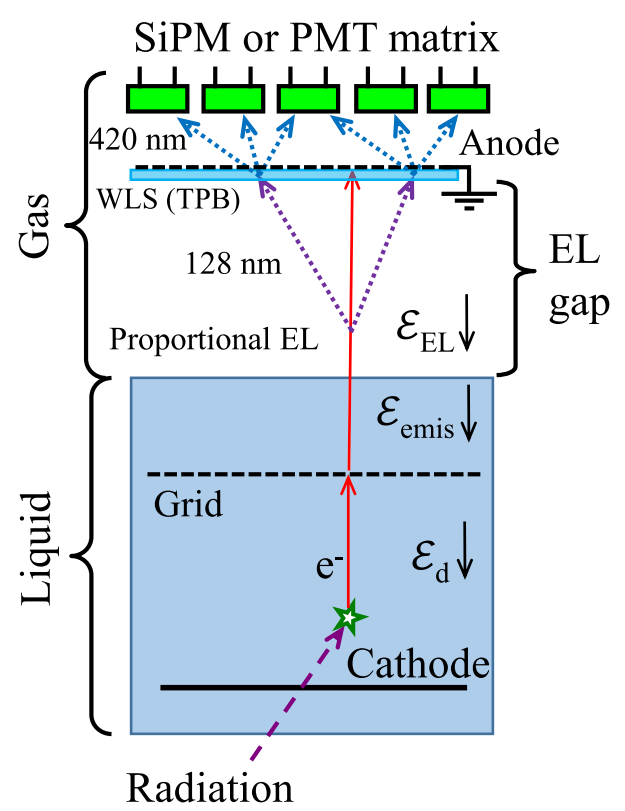

Fig. 3 "Standard" concept of SiPM-matrix readout of two-phase argon detectors with an EL gap

WLS degradation and its dissolving in liquid Ar [12], as well as that of WLS peeling off from the substrate.

In the first alternative concept, hereinafter referred to as "direct SiPM-matrix readout", the detection threshold for S2 signal might increase compared to that of the "standard" concept at higher electric fields (exceeding $5 \mathrm{Td}$ ), since here the light yield of $\mathrm{NBrS}$ electroluminescence is lower compared to that of ordinary electroluminescence: see Fig. 2. On the other hand, for lower reduced electric fields, between 4 and $5 \mathrm{Td}$, the response of PMTs and SiPMs to NBrS electroluminescence might be comparable with that of ordinary electroluminescence recorded using a WLS [15]. This is because the $\mathrm{NBrS}$ EL is recorded mostly directly thanks to its spectrum part in the visible range, and thus practically without losses, while ordinary EL is recorded indirectly, with significant reduction of the photon flux after re-emission by the WLS, the reduction factor reaching 15-20 [15] (in the absence of optical contact between the WLS and SiPM). Note that the EL field employed in the DarkSide-50 search for low-mass WIMPs in an S2-only mode was $4.2 \mathrm{kV} / \mathrm{cm}$, corresponding to $4.8 \mathrm{Td}$ [4].

In the second alternative concept, hereinafter referred to as "THGEM/SiPM-matrix readout", an additional charge amplification of the S2 signal is provided by applying a voltage across the THGEM, resulting in electron avalanching in THGEM holes. Accordingly, the SiPM matrix records avalanche scintillations in the NIR from the THGEM holes, rather than electroluminescence from the EL gap. In this case, the detection threshold for the S2 signal can be significantly decreased, compared to direct SiPM-matrix readout.
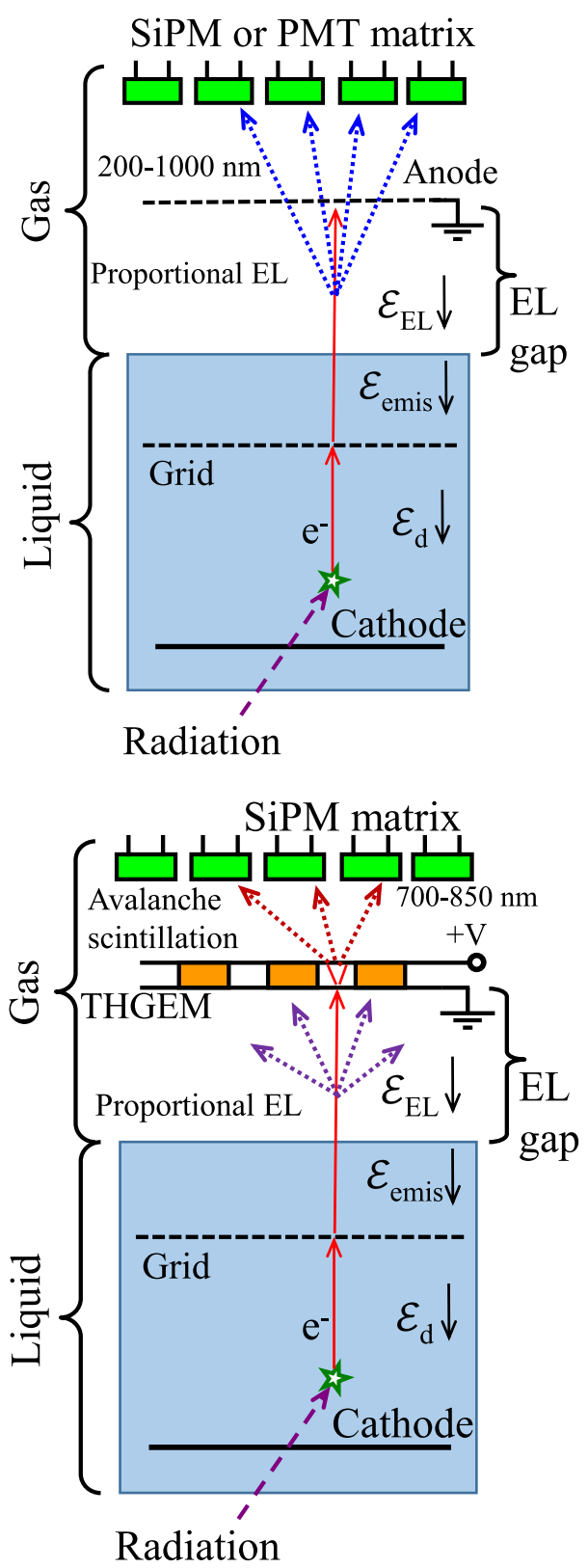

Fig. 4 Two alternative concepts of SiPM-matrix readout of two-phase argon detectors with EL gap proposed elsewhere [14,15] and experimentally studied in the present work: that of SiPM matrix directly coupled to EL gap ("direct SiPM-matrix readout") (top) and that of combined THGEM/SiPM-matrix multiplier coupled to EL gap ("THGEM/SiPM-matrix readout") (bottom)

It should be remarked that the concept of THGEM/SiPMmatrix readout overlaps with the earlier idea of Cryogenic Avalanche Detectors (CRADs), developed elsewhere [7,26, 27]. In CRADs, the charge multiplication or avalanche scintillation signal from the THGEM (or GEM), placed in the gas phase of the two-phase detector, is recorded. The difference is that in the CRAD concept, the gas gap underneath the THGEM is not supposed to operate in EL mode (i.e. it operates at relatively low electric fields). In contrast, in 
Table 1 Characteristic properties of three SiPMs types used in SiPM matrices

\begin{tabular}{llll}
\hline SiPM type (producer) & Active area $\left(\mathrm{mm}^{2}\right)$ & Number of pixels & Active area fill factor $(\%)$ \\
\hline MRS APD 149-35 (CPTA) & $2.1 \times 2.1$ & 1764 & 62 \\
MPPC S10931-100P (Hamamatsu) & $3 \times 3$ & 900 & 78.5 \\
MPPC S13360-6050PE (Hamamatsu) & $6 \times 6$ & 14400 & 74 \\
\hline
\end{tabular}

THGEM/SiPM-matrix readout concept, the EL gap is needed to record proportional electroluminescence in addition to that of avalanche scintillation, using either the bottom or side SiPM matrices, to provide the superior amplitude resolution when recording single drifting electrons. This is needed because in these conditions the amplitude resolution of the THGEM is not sufficient: it is significantly deteriorated due to intrinsic fluctuations of the electron avalanche [28].

In the following sections, we first describe the development of SiPM matrices for operation in two-phase argon detectors, and then report the implementation of these alternative readout concepts in our experimental setup.

\section{R \& D of SiPM matrices operated in two-phase argon detectors}

In the course of this study, three SiPM matrices were progressively developed for operation in two-phase argon detectors, with a channel pitch of $1 \mathrm{~cm}$ and matrix size of $5 \times 5$ of active channels. Three different types of SiPMs were used in the matrices, respectively: see Table 1 .

The first SiPM type was MRS APD 149-35 (CPTA) [29]: see Fig. 5a. At $87 \mathrm{~K}$, it showed an acceptable dark count rate (about $6 \mathrm{~Hz} / \mathrm{mm}^{2}$ ) with a gain of about $10^{6}$ [30]. However, during the first cryogenic run, half of the 25 channels failed, making impossible further use of the matrix.

The second SiPM type was MPPC S10931-100P (Hamamatsu) [23]: see Figs. 5b, 6 and 7). At 87 K, it had a lower dark count rate (about $0.6 \mathrm{~Hz} / \mathrm{mm}^{2}$ ) and half as much maximum gain $\left(5 \cdot 10^{5}\right)$ with respect to MRS APD 149-35 [31]. The $5 \times 5 \mathrm{SiPM}$ matrix made from these SiPMs demonstrated stable operation for more than 20 cooling/heating cycles. However, this SiPM type has a narrow operating voltage range, resulting in substantial gain variations from channel to channel when powered by the same voltage.

The third (most successful) SiPM type was MPPC S13360-6050PE (Hamamatsu) [23]: see Figs. 5c, 6 and 7. At $87 \mathrm{~K}$, it demonstrated a low dark count rate (about $0.1 \mathrm{~Hz} / \mathrm{mm}^{2}$ ) and high gains reaching $9 \cdot 10^{6}$ (these characteristics were measured following the procedure described in $[30,31])$. In addition, the MPPC S13360-6050P has a lower operating voltage and wider voltage range compared to MPPC S10931-100P, which significantly facilitated its use.

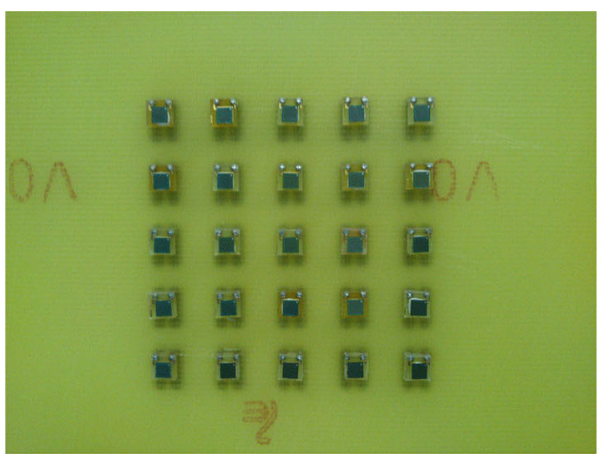

(a)

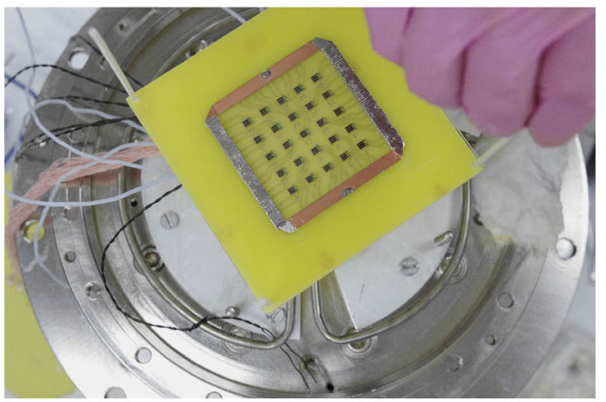

(b)

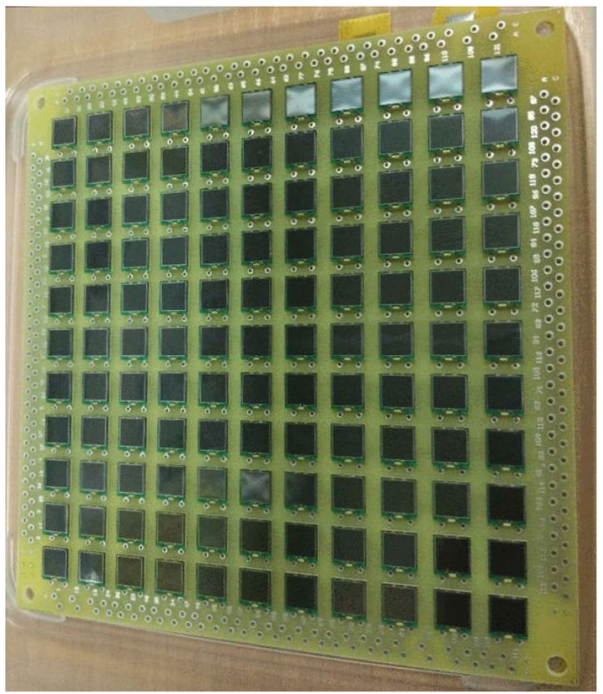

(c)

Fig. 5 Photographs of SiPM matrices progressively developed in this work. (a) $5 \times 5 \mathrm{SiPM}$ matrix made from MRS APD 149-35 (CPTA) with an active area of $2.1 \times 2.1 \mathrm{~mm}^{2}$. (b) $5 \times 5 \mathrm{SiPM}$ matrix made from MPPC S10931-100P (Hamamatsu) with an active area of $3 \times 3 \mathrm{~mm}^{2}$. (c) $11 \times 11$ SiPM matrix made from MPPC S13360-6050PE (Hamamatsu) with an active area of $6 \times 6 \mathrm{~mm}^{2}$. The SiPM channel pitch is $1 \mathrm{~cm}$ in all cases 


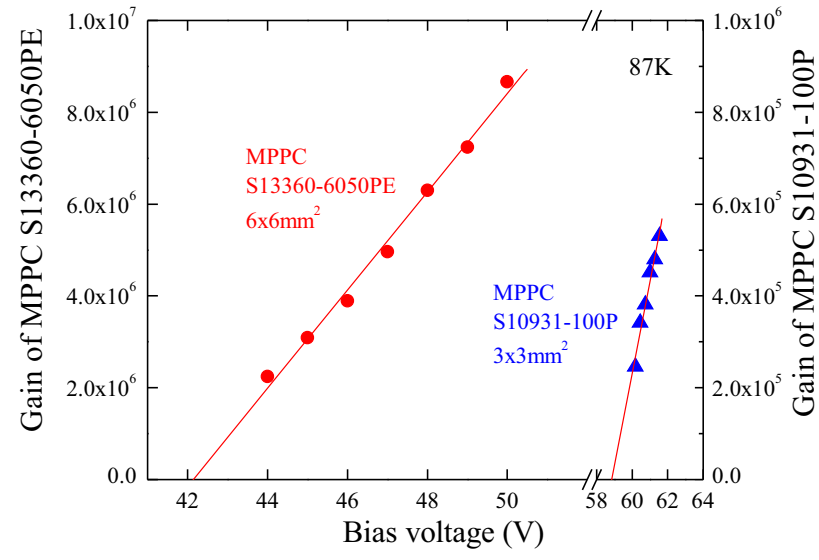

Fig. 6 Gain-voltage characteristics of different SiPM types at $87 \mathrm{~K}$

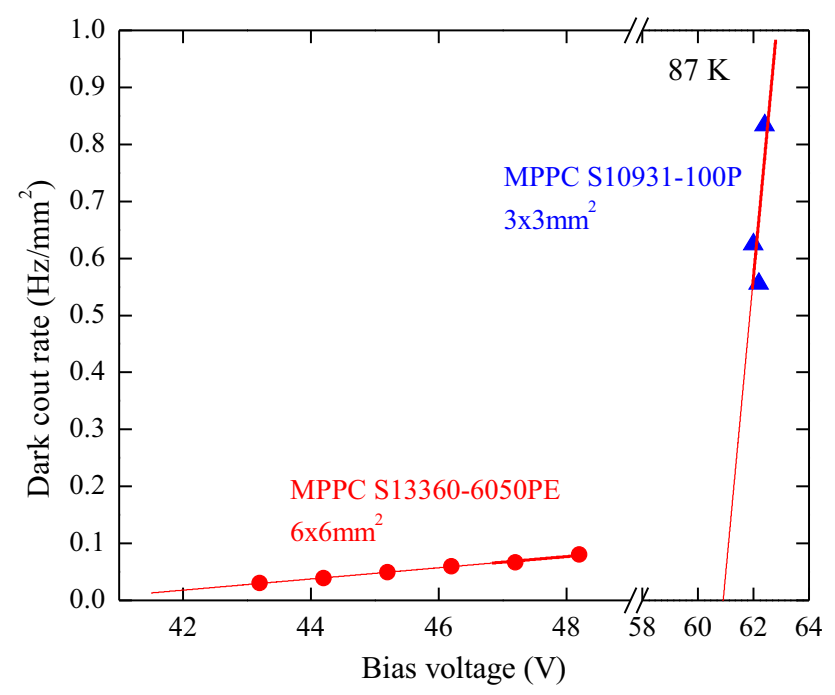

Fig. 7 Dark count rates of different SiPM types as a function of the bias voltage at $87 \mathrm{~K}$

The real matrix size was $11 \times 11$ channels, of which only the central part of $5 \times 5$ channels was active in the current measurements. This SiPM matrix demonstrated stable operation over 30 cooling/heating cycles and still is being used in our experimental setup.

\section{Experimental setup}

Figure 8 shows the experimental setup of the Novosibirsk group of the DarkSide collaboration. It included a 9-1 cryogenic chamber filled with 2.51 of liquid argon. The detector was operated in a two-phase mode in the equilibrium state at a saturated vapor pressure of $1.00 \mathrm{~atm}$ and temperature of $87.3 \mathrm{~K}$. Argon, of initial purity of $99.998 \%$, was additionally purified from electronegative impurities during each cooling cycle by an Oxisorb filter, providing electron life-time in the liquid exceeding $100 \mu \mathrm{s}$ [32].

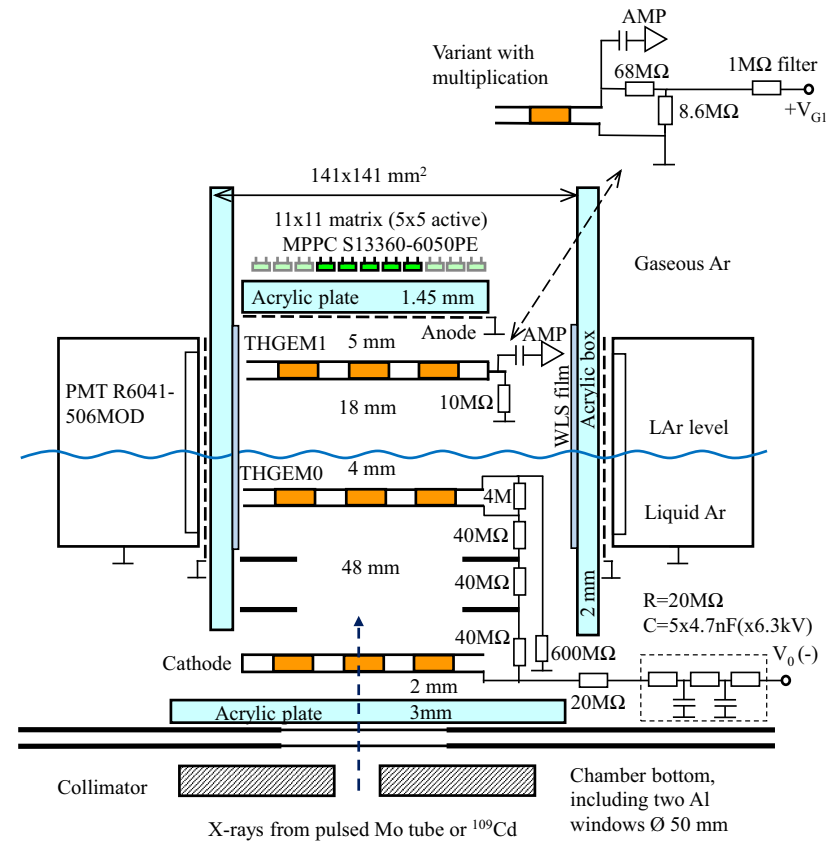

Fig. 8 Schematic view of the experimental setup. The electric fields lines in the TPC were presented elsewhere [34]

The detector was a two-phase LAr time-projection chamber (TPC) composed of the drift (48 mm thick) and electron emission (4 mm thick) regions, in the liquid phase, and the EL gap (18 $\mathrm{mm}$ thick), in the gas phase. To form these regions, we used THGEM (Thick Gas Electron Multipliers, [33]) electrodes instead of the more conventional wire grids, providing the advantage of electrode rigidity that allowed to avoid wire grid sagging. All electrodes had the same active area of $10 \times 10 \mathrm{~cm}^{2}$. The THGEM geometrical parameters were the following: dielectric (FR-4) thickness of $0.4 \mathrm{~mm}$, hole pitch of $0.9 \mathrm{~mm}$, hole diameter of $0.5 \mathrm{~mm}$ and hole rim of $0.1 \mathrm{~mm}$, optical transparency at normal incidence of $28 \%$.

The drift region was formed by a cathode electrode, field-shaping electrodes and THGEM0 (interface THGEM), immersed in the liquid layer. These were biased through a resistive high-voltage divider placed within the liquid. THGEM0 was biased in a way to provide a transmission of drifting electrons from the drift region to that of electron emission: the electrons drifted successively from a lower to higher electric field region. The electron transmission efficiency, defined by the voltage applied across THGEM0 and its geometrical parameters, was calculated in [34] to be $62 \%$.

THGEM1 was placed in the gas phase above the liquid and acted either as an anode of the EL gap (grounded through a resistor) or an electron multiplication element of the combined THGEM/SiPM-matrix multiplier (i.e. operated in electron avalanche mode), coupled to the EL gap.

The liquid level in the EL gap was monitored with an accuracy of $0.5 \mathrm{~mm}$, being calculated from the amount of con- 
densed Ar using a computer-aided design (CAD) software, the latter verified in special calibration runs with THGEM1 operated as a capacitive liquid level meter [35].

Three different readout configurations, corresponding to three EL mechanisms, were used in the measurements. In the first configuration, based on the ordinary EL mechanism, the EL gap was viewed by four compact cryogenic PMTs R6041-506MOD [36], located on the perimeter of the gap and electrically insulated from it by an acrylic box. Three of four PMTs were made sensitive to the VUV via WLS films (based on TPB in a polystyrene matrix [37]) deposited on the inner box surface facing the EL gap, in front of these PMTs. We designate this configuration as 3PMT + WLS.

The second readout configuration corresponds to the concept of direct SiPM-matrix readout (see Fig. 4), based on the NBrS EL mechanism. Here the SiPM matrix, placed in the gas phase, is directly coupled to the EL gap, with light reaching the SiPMs through the holes of THGEM1 and the acrylic plate, the latter being used as additional electrical insulation. The SiPM matrix (see Fig. 5c) was made from MPPCs 13360-6050PE [23] operated at an overvoltage of 5.6 V; their properties were described in the previous section. Taking into account the transmission of the acrylic plate in front of the matrix (see Fig. 2), the SiPM matrix sensitivity ranges from the near UV $(360 \mathrm{~nm})$ to the NIR $(1000 \mathrm{~nm})$. The contribution of crosstalk from the VUV, re-emitted by WLS on the side walls to the signal recorded by the SiPM matrix, was negligible as shown by experiments under similar conditions without WLS.

The third configuration corresponds to the concept of THGEM/SiPM-matrix readout (see Fig. 4), based on the avalanche scintillation mechanism. Here the combined THGEM/SiPM-matrix multiplier is coupled to the EL gap. In this case, a voltage is applied across THGEM1 (see top part of Fig. 8). In addition to avalanche scintillations in the NIR, the SiPM matrix also recorded NBrS electroluminescence from the EL gap; its contribution however was negligible (of about $3 \%$ at THGEM1 charge gain of 37).

It should be remarked that the detector was optimized for studying the all three readout techniques in the same experimental setup, rather than for reaching the maximum light yields. In particular for direct SiPM-matrix readout, the THGEM1 electrode acted as an optical mask, significantly (nine times) reducing the light flux: first, due to optical transparency at normal incidence, of $28 \%$, and, second, due to angle dependence factor for optical transmission, of $40 \%$ (determined by Monte-Carlo simulation). This, however, does not prevent in the following to assess the maximum light yields and detection thresholds that would be achieved under optimal conditions.

The detector was irradiated from outside either by X-rays from a pulsed X-ray tube with Mo anode, with an average deposited energy in liquid $\mathrm{Ar}$ of $25 \mathrm{keV}$ [38], or by gamma rays from a ${ }^{109} \mathrm{Cd}$ source [39]. To study the position resolution of the detector, a narrow beam of gamma-rays and $\mathrm{X}$-rays was provided by a collimator with a hole diameter of $2 \mathrm{~mm}$.

The signals from the PMTs were amplified using fast 10fold amplifiers CAEN N979 and then re-amplified with linear amplifiers with a shaping time of $200 \mathrm{~ns}$. The signals from 3PMT + WLS were summed (using CAEN N625 unit). The signals from each SiPM were transmitted to amplifiers with a shaping time of $40 \mathrm{~ns}$, via twisted pair wires. The charge signal from THGEM1 was recorded using a calibrated chain of a preamplifier and a shaping amplifier. All amplifiers were placed outside the two-phase detector.

The SiPM signal amplitude was defined in terms of the number of recorded photoelectrons. The contribution of SiPM crosstalk (between the pixels) was accounted for and subtracted accordingly. One of the channels of the SiPM matrix was inactive during data acquisition and the photoelectron number in it was determined as the average of two adjacent channels (see Fig. 19).

The DAQ system included both a 4-channel oscilloscope, model LeCroy WR HRO 66Zi, and a 64-channel Flash ADC CAEN V1740 (12 bits, $62.5 \mathrm{MHz}$ ): the signals were digitized and stored both in the oscilloscope and in a computer for further off-line analysis. Other details of the experimental setup and measurement procedures can be found elsewhere $[15,40]$.

\section{EL gap yield for direct SiPM-matrix readout}

The performance of the two-phase detector with direct SiPMmatrix readout is characterized by the EL gap yield. It is defined as the number of photoelectrons (PE) recorded by the SiPM matrix in total per drifting electron in the EL gap.

To measure the EL gap yield, a ${ }^{109} \mathrm{Cd}$ gamma-ray source was used. The emission spectrum of this source includes lowenergy (22-25 keV) and high-energy lines: namely the characteristic lines of $\mathrm{W}(59 \mathrm{keV})$, which was used as a radionuclide substrate, and the $88 \mathrm{keV}$ line of ${ }^{109} \mathrm{Cd}$ itself [39]. Due to insufficient energy resolution, the 59 and $88 \mathrm{keV}$ lines could not be separated; therefore their weighted average energy (82 keV [39]) was used in the analysis.

Due to the small photoelectron number, it was not possible to directly separate the low and high energy parts in the SiPM amplitude spectrum: see Fig. 9. On the other hand, the 3PMT+WLS amplitude was high enough to make such a separation: see Fig. 10.

Since the 3PMT+WLS and SiPM-matrix signals are correlated (see Fig. 11), it is possible to separate the events with higher and lower energy in the SiPM-matrix amplitude spectrum, selecting appropriately the events in the 3PMT+WLS amplitude spectrum. This is seen in Fig. 9 showing the 


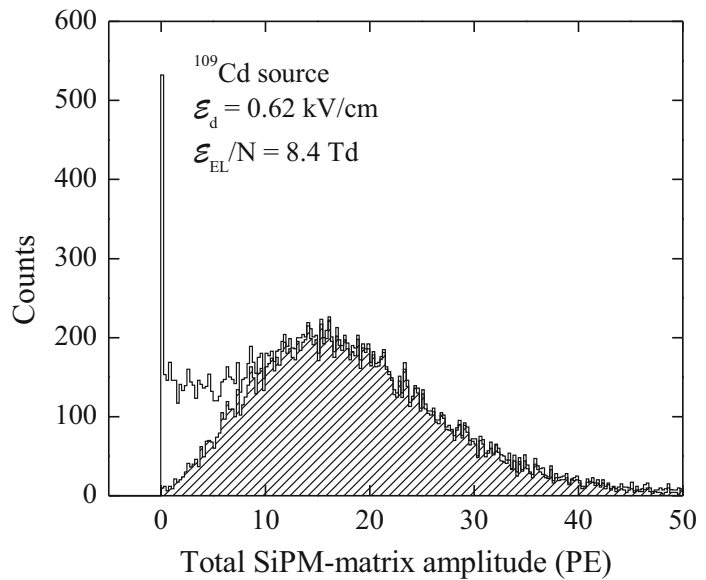

Fig. 9 Direct SiPM-matrix readout: amplitude spectrum of the total SiPM-matrix signal obtained with ${ }^{109} \mathrm{Cd}$ source. The hatched area corresponds to the higher energy peak of the 3PMT + WLS signals (see Fig. 10)

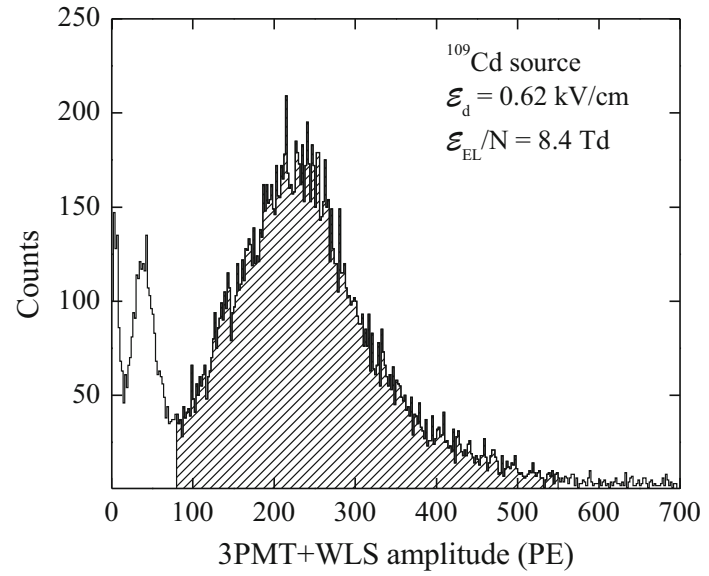

Fig. 10 Direct SiPM-matrix readout: amplitude spectrum of the total 3PMT + WLS signal obtained with ${ }^{109} \mathrm{Cd}$ source for the maximum field in the EL gap. The higher energy peak, corresponding to $59-88 \mathrm{keV}$ gamma-rays, is hatched

SiPM-matrix amplitude spectrum, where the hatched area is obtained by selecting the 3PMT+WLS signals from the higher energy peak: see Fig. 10. Only the average photoelectron number of this (high-energy) part of the spectrum was used to determine the EL gap yield.

In addition, to calculate the EL gap yield, one has to know the charge emitted from the liquid into the EL gap. Since it was too small for direct recording (about $800 \mathrm{e}^{-}$), it was calculated theoretically using the data on ionization yields for electron recoils in liquid argon [38] and on electron transmission through the THGEM0 electrode [34].

The EL gap yield was obtained by dividing the average photoelectron number recorded by the SiPM matrix in total to the calculated charge. The EL gap yield obtained this way, as a function of the electric field in the EL gap, is shown

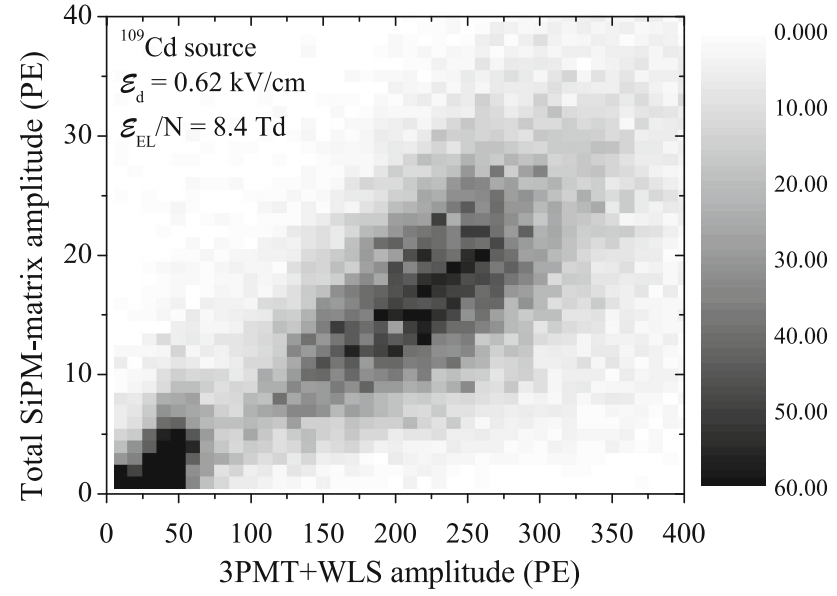

Fig. 11 Direct SiPM-matrix readout: correlation between the amplitude of the total SiPM-matrix and 3PMT + WLS signals

Reduced electric field (Td)

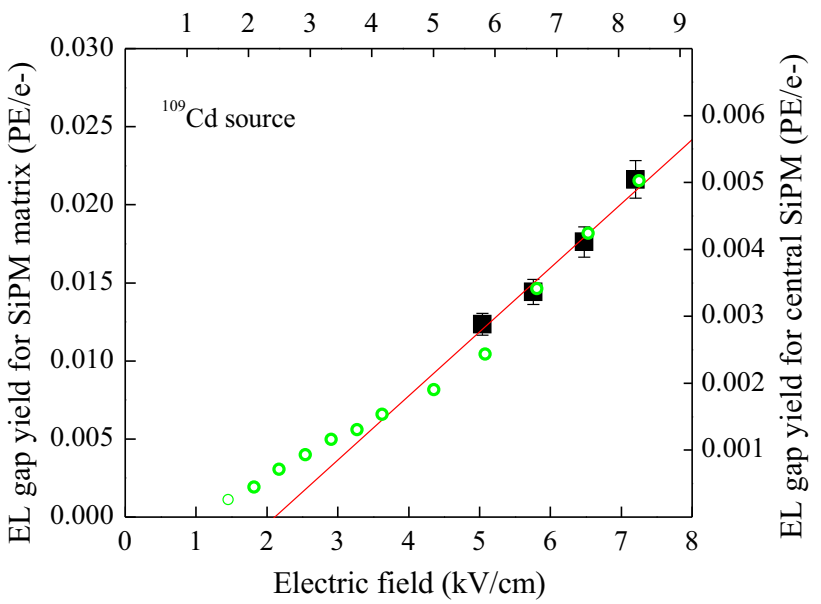

Fig. 12 Direct SiPM-matrix readout: EL gap yield for the SiPM matrix in total (solid data points, left scale) as a function of the electric field or reduced electric field in the EL gap, at the average energy of $82 \mathrm{keV}$ deposited by gamma-rays from ${ }^{109} \mathrm{Cd}$ source in liquid Ar. The red line is a linear fit to the data points. For comparison, the EL gap yield for the central SiPM measured elsewhere [16] is shown (open data points, right scale)

in Fig. 12. At higher fields, between 5 and $8 \mathrm{Td}$, the field dependence is well described by a linearly growing function. For comparison, the EL gap yield for the central SiPM only, measured for wider field range in our previous work [16], is shown. One can see a good reproducibility of the linear field dependence in data overlap.

The maximum EL gap yield amounted to $0.022 \mathrm{PE} / \mathrm{e}^{-}$at an electric field in the EL gap of $7.3 \mathrm{kV} / \mathrm{cm}$ (corresponding to the reduced field of $8.4 \mathrm{Td}$ ), which corresponds to $0.2 \mathrm{PE}$ per $\mathrm{keV}$ of the energy deposited in liquid Ar. This value is fairly small. We will see in the following (Sect. 9) that it can be significantly increased, by about an order of magnitude, for the optimal detector structure. 


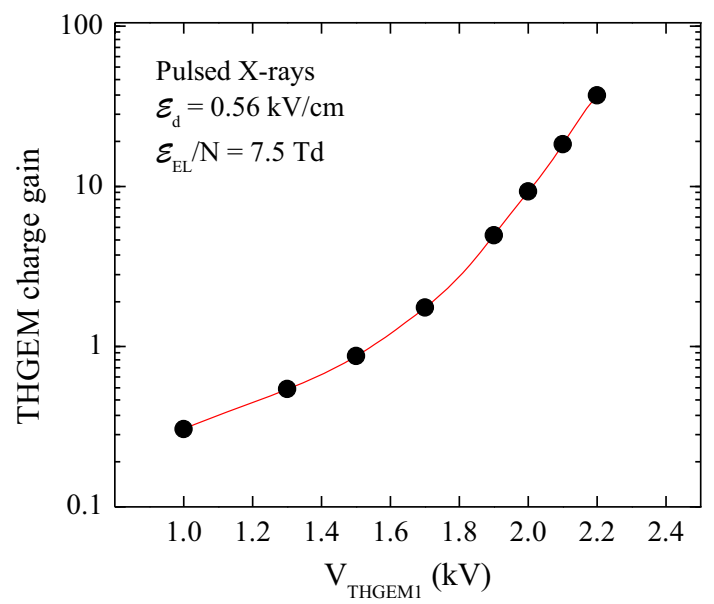

Fig. 13 THGEM/SiPM-matrix readout: charge gain of the THGEM1 multiplier as a function of the voltage across it, at fixed electric fields in the drift and EL regions

\section{THGEM/SiPM-matrix yield}

Similarly to the EL gap yield with direct SiPM-matrix readout, we can define the yield of the combined THGEM/SiPMmatrix multiplier coupled to the EL gap (or THGEM/SiPMmatrix yield for short), as the number of photoelectrons recorded by the SiPM matrix per drifting electron in the EL gap.

Here, THGEM1 was operated in electron avalanche mode, its charge gain being measured using a pulsed X-ray tube (similarly to [41]). Figure 13 shows THGEM1 charge gain as a function of the voltage applied across it, at fixed drift and EL gap electric fields.

The yield of the combined THGEM/SiPM-matrix multiplier was measured at two THGEM1 voltages, of 2.0 and $2.2 \mathrm{kV}$, corresponding to THGEM1 charge gain of 9 and 37 . Using the ${ }^{109} \mathrm{Cd}$ source, the amplitude spectra of the signals from the SiPM matrix were recorded. Due to sufficient energy resolution, it was possible to separate the low-energy (22$25 \mathrm{keV}$ ) and high-energy events (59-88 keV) without using the 3PMT+WLS signals: see Fig. 14. It should be remarked that the degradation of energy resolution of the combined THGEM/SiPM-matrix multiplier due to inherent avalanche fluctuations, compared to the direct SiPM-matrix readout, becomes insignificant at higher statistics of drifting electrons (exceeding 10) due to multiple spectrum convolution.

Similarly to direct SiPM-matrix readout, the average number of photoelectrons for the high-energy part of the spectrum was defined and then divided by the calculated charge emitted into the EL gap. The THGEM/SiPM-matrix yield obtained this way is shown in Fig. 15. One can see that the yield is sensitive to the THGEM gain, rather than to the electric field in the EL gap. This is because the THGEM/SiPM-matrix yield,

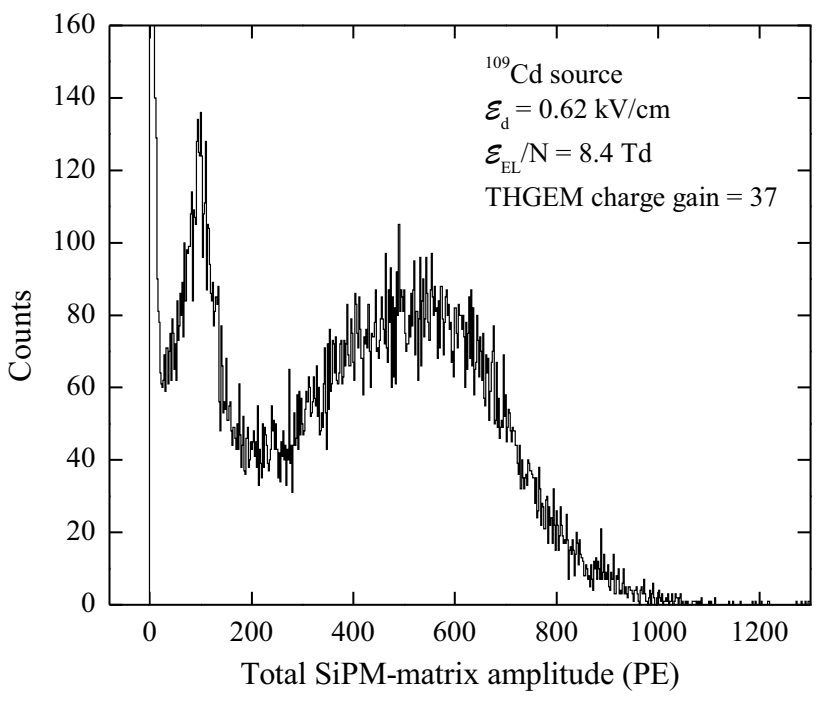

Fig. 14 THGEM/SiPM-matrix readout: amplitude spectrum of the total SiPM-matrix signal obtained with ${ }^{109} \mathrm{Cd}$ source, at THGEM1 charge gain of 37

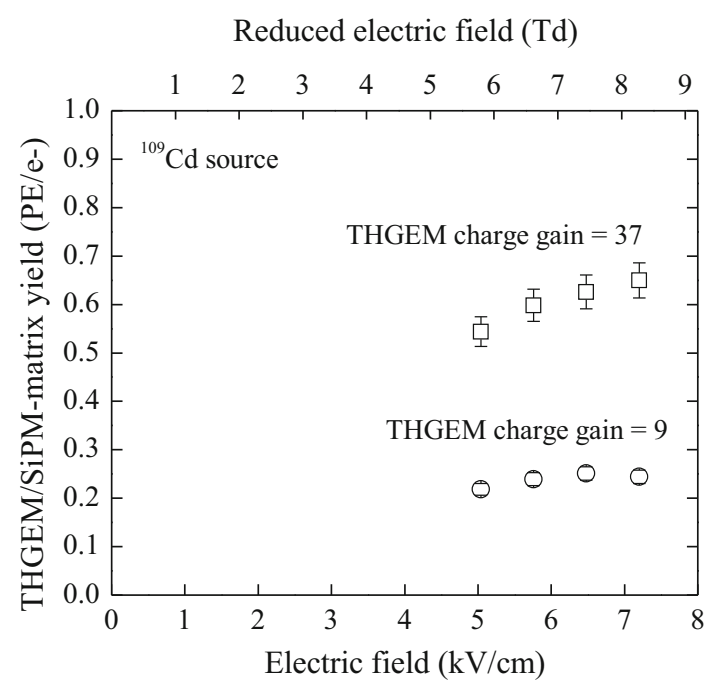

Fig. 15 THGEM/SiPM-matrix readout: THGEM/SiPM-matrix yield as a function of the electric field or reduced electric field in the EL gap at the average energy of $82 \mathrm{keV}$, deposited by gamma-rays from ${ }^{109} \mathrm{Cd}$ source in liquid argon, measured at two THGEM charge gains

being first of all proportional to the THGEM charge gain, weakly depends on the external electric field.

The maximum THGEM/SiPM-matrix yield amounted to $0.65 \mathrm{PE} / \mathrm{e}^{-}$at a charge gain of 37 and electric field in the EL gap of $7.3 \mathrm{kV} / \mathrm{cm}$, which corresponds to $6.2 \mathrm{PE}$ per $\mathrm{keV}$ of the energy deposited in liquid Ar. One can see that even at such a moderate THGEM gain, the amplitude yield of the THGEM/SiPM-matrix readout is considerably (by more than order of magnitude) increased compared to the direct SiPMmatrix readout. In Sect. 9, we will estimate the detection 
thresholds for nuclear recoils for these readout techniques, under the optimal conditions.

\section{$7 x, y$ coordinate reconstruction algorithm}

One of the main advantages of the SiPM matrix readout is the high reconstruction accuracy of the event coordinates in $x, y$ plane of the two-phase detector. In this section and the next one, the reconstruction algorithm and the position resolution will be described. These results were obtained in the twophase detector when irradiated by a pulsed X-ray tube or ${ }^{109} \mathrm{Cd}$ source through a $2 \mathrm{~mm}$ collimator.

Let us define the following values: $x_{0}$ is the true coordinate of the X-ray photon interaction point in the liquid, $X_{i}$ is the coordinate of the center of the $i$ th element of the SiPM matrix, $N_{i}$ is the number of photoelectrons recorded by the $i$ th element of the SiPM matrix, $N_{c h}$ is the number of channels of the SiPM matrix, $x_{\text {exp }}$ and $x_{\text {sim }}$ are coordinates of the interaction point reconstructed from experimental data and simulation, respectively.

The center of gravity (CoG) algorithm is one of the simplest methods widely used for coordinate reconstruction [42]. According to this algorithm, $x_{\text {exp }}$ is calculated using the following formula:

$x_{\text {exp }}=\left(\sum_{i=1}^{N_{c h}} X_{i} \cdot N_{i}\right) /\left(\sum_{i=1}^{N_{c h}} N_{i}\right)$.

Similar formulas are used for $y$ coordinate.

A well-known feature of the CoG algorithm is the compression effect, resulting in that the reconstructed coordinates are biased to the center of the matrix [43]. To eliminate such a systematic bias, it is necessary to find the dependence of the reconstructed coordinate on the true one: $x_{\exp }\left(x_{0}\right)$. Since in our case $x_{0}$ is not known from experimental data, the desired dependence $x_{\text {exp }}\left(x_{0}\right)$ is determined by simulation: $x_{\text {sim }}\left(x_{0}\right)$ and its inverse function $x_{0}\left(x_{\text {sim }}\right)$ [44].

To find these dependences, it is obviously necessary to know how detected photons (i.e. photoelectrons) are distributed over the elements of the SiPM matrix for the given coordinates of the interaction point $\left(x_{0}, y_{0}\right)$. This distribution over the elements of the SiPM matrix $\left(N_{i}\right)$ is described by the following expression:

$N_{i}\left(x_{0}, y_{0}\right)=N_{0} \cdot L R F_{i}\left(X_{i}-x_{0}, Y_{i}-y_{0}\right)$,

where $N_{0}$ is the number of photons emitted at the interaction point $\left(x_{0}, y_{0}\right)$, and $L R F_{i}$ is the so-called Light Response Function [45], i.e. the fraction of photons registered by $i$-th element of the SiPM matrix for a given interaction point $\left(x_{0}\right.$, $y_{0}$ ). It is obvious that $L R F_{i}$ has a maximum when $X_{i}-x_{0}=$

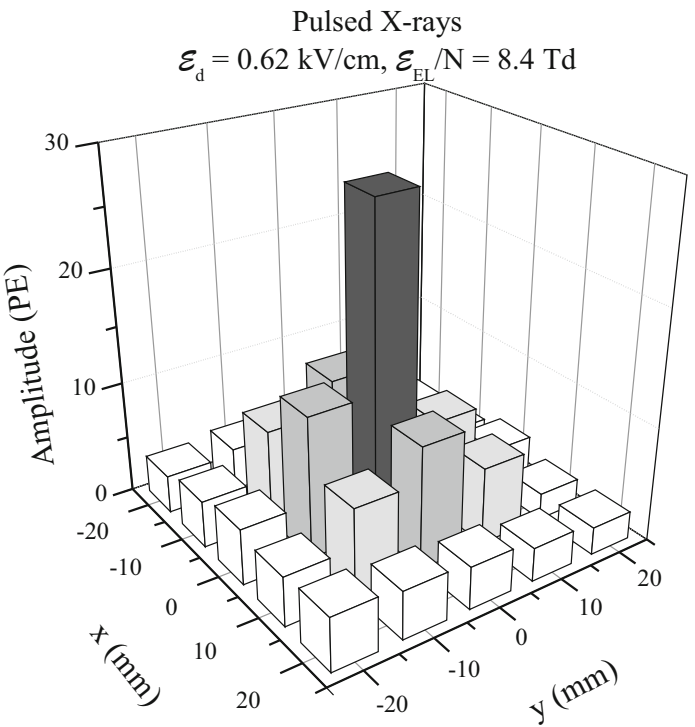

Fig. 16 Direct SiPM-matrix readout: the averaged distribution of photoelectrons over the SiPM-matrix channels in X,y plane for "central" events, in which the distribution's maximum is at the central channel. The data were obtained at the maximum reduced EL field, of $8.4 \mathrm{Td}$, when the detector was irradiated by pulsed X-rays

$Y_{i}-y_{0}=0$, i.e. when the projection of the interaction point is in the center of the channel.

In principle, $L R F_{i}$ can be calculated by Monte Carlo (MC), simulating the propagation of photons in the detector. However, this is a difficult task, since the correct description of the properties of all optical surfaces is not always achievable. In this regard, $L R F_{i}$ is determined empirically, from experimental data.

To determine $L R F_{i}$, first of all, an averaged distribution of photoelectrons $N_{i}$ over the channels of the SiPM matrix for "central" events (for which the maximum of the distribution hits the central channel) was obtained. Figure 16 shows 3D distribution and Fig. 17 its 2D cross-sections for such "central" events.

Next, we use the approximation that the $L R F_{i}$ shape is the same for all SiPM-matrix channels. This approximation is justified by the fact that the interaction region in $x, y$ plane was much smaller (less than $0.5 \mathrm{~cm}$ in diameter) than the active region of the detector $\left(10 \times 10 \mathrm{~cm}^{2}\right)$. Thus, the $L R F$ obtained for the central channel could be used for all other SiPM-matrix channels. The $L R F$ (up to scaling factor) was found from Fig. 16 using a linear interpolation.

Using the $L R F$ obtained this way, $x_{0}\left(x_{s i m}\right)$ and $y_{0}\left(y_{s i m}\right)$ dependencies were found. To this end, $x_{0}$ and $y_{0}$ coordinates were randomly and uniformly generated in the range of $(-20 \mathrm{~mm}, 20 \mathrm{~mm})$, and then $x_{\text {sim }}$ and $y_{\text {sim }}$ coordinates were calculated using the $\mathrm{CoG}$ formula (1). Figure 18 shows the resulting $x_{0}\left(x_{\text {sim }}\right)$ dependence obtained this way, along 

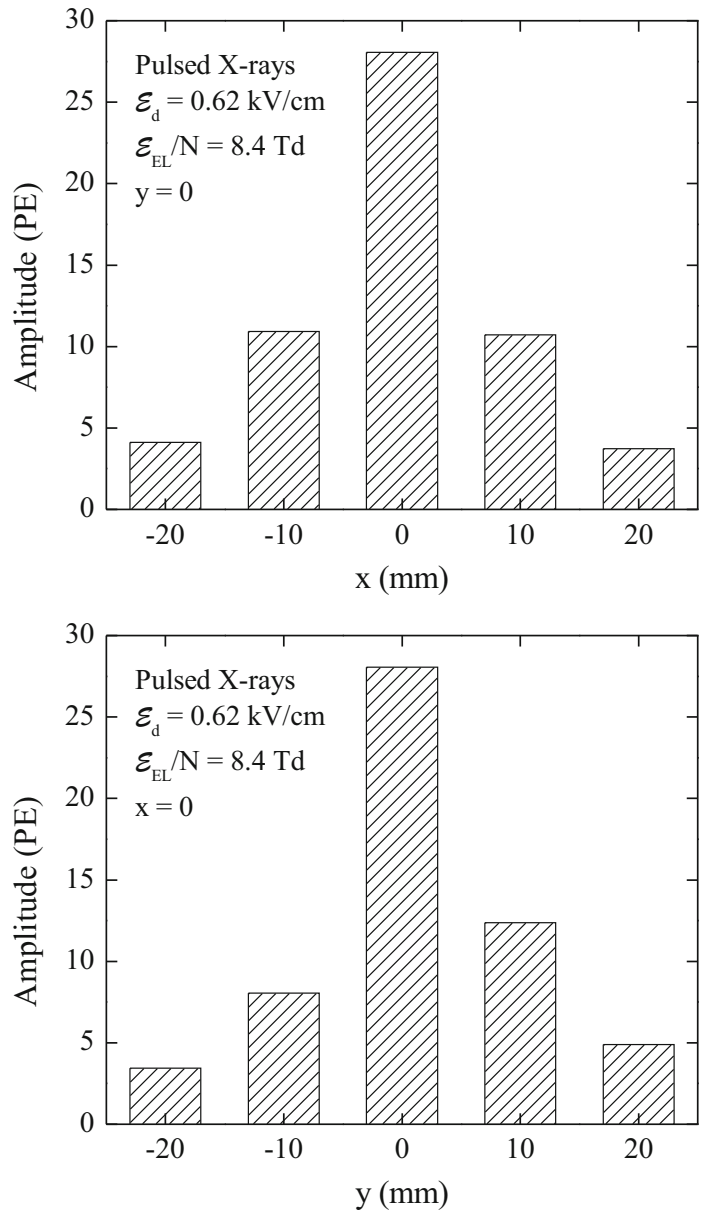

Fig. 17 Direct SiPM-matrix readout: 2D cross-section of Fig. 16 at $y$ $=0$ (top) and $x=0$ (bottom)

with the trivial $x_{0}=x_{\text {sim }}$ dependence (i.e. in the absence of systematic bias).

\section{Results of $x, y$ reconstruction}

Applying the $\mathrm{CoG}$ algorithm to the experimental data and taking into account the corrections for a systematic bias using the fitted curve in Fig. 18, the desired event distributions over $x_{0}\left(x_{\text {exp }}\right)$ and $y_{0}\left(y_{\text {exp }}\right)$ were obtained. In particular, Fig. 19 shows $2 \mathrm{D}$ distribution of the event coordinates in $x_{0}\left(x_{\text {exp }}\right)$, $y_{0}\left(y_{\text {exp }}\right)$ plane for direct SiPM-matrix readout, when the detector was irradiated by pulsed X-rays through a $2 \mathrm{~mm}$ collimator.

Figure 20 shows the projections of Fig. 19 on $x$ and $y$ axes. The fit of the distribution on $x_{0}\left(x_{\text {exp }}\right)$ and $y_{0}\left(y_{\text {exp }}\right)$ (red curve) and the rectangular distribution of the true coordinate of the interaction point (blue dotted curve) are also shown. The latter was determined geometrically taking into account the relative position of the radiation source and collimator and the X-ray range in liquid Ar. The fit function represented

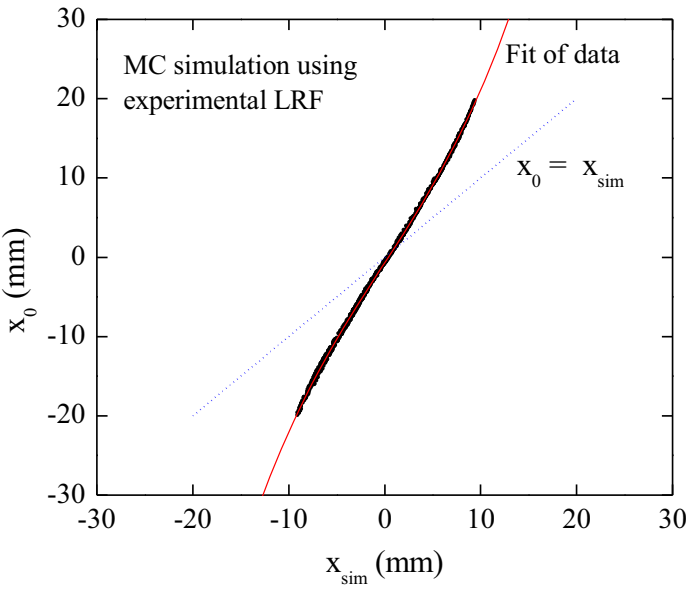

Fig. 18 Direct SiPM-matrix readout: true coordinate of interaction point $x_{0}$ as a function of reconstructed coordinate $x_{\text {sim }}$ (black dots), the latter obtained using MC simulation with the experimental $L R F$, and a fit of this dependence by polynomial function (red curve). For comparison, the dependence $x_{0}=x_{\text {sim }}$ is shown (blue dotted line)

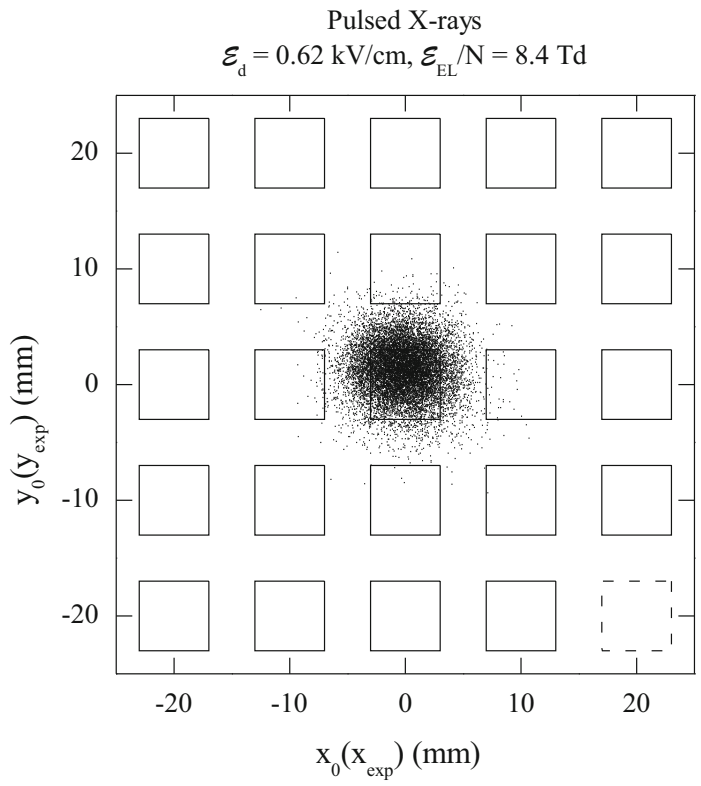

Fig. 19 Direct SiPM-matrix readout: 2D coordinate distribution of reconstructed events in $x_{0}\left(x_{\text {exp }}\right), y_{0}\left(y_{\text {exp }}\right)$ plane. The solid boxes are the active SiPMs and the dashed box is the inactive SiPM (the photoelectron number in which was determined as the average of two adjacent channels). The data were obtained at the maximum reduced EL field, of $8.4 \mathrm{Td}$, when the detector was irradiated by pulsed X-rays through a $2 \mathrm{~mm}$ collimator

a convolution of this rectangular distribution with a Gaussian function. The latter is defined by the detector resolution. Thus, the fitting parameter of the Gauss function $(\sigma)$ characterizes the position resolution of the detector.

Figure 21 shows an example of the amplitude spectrum of the total SiPM-matrix signal, at the maximum EL field (compare to Fig. 9). 

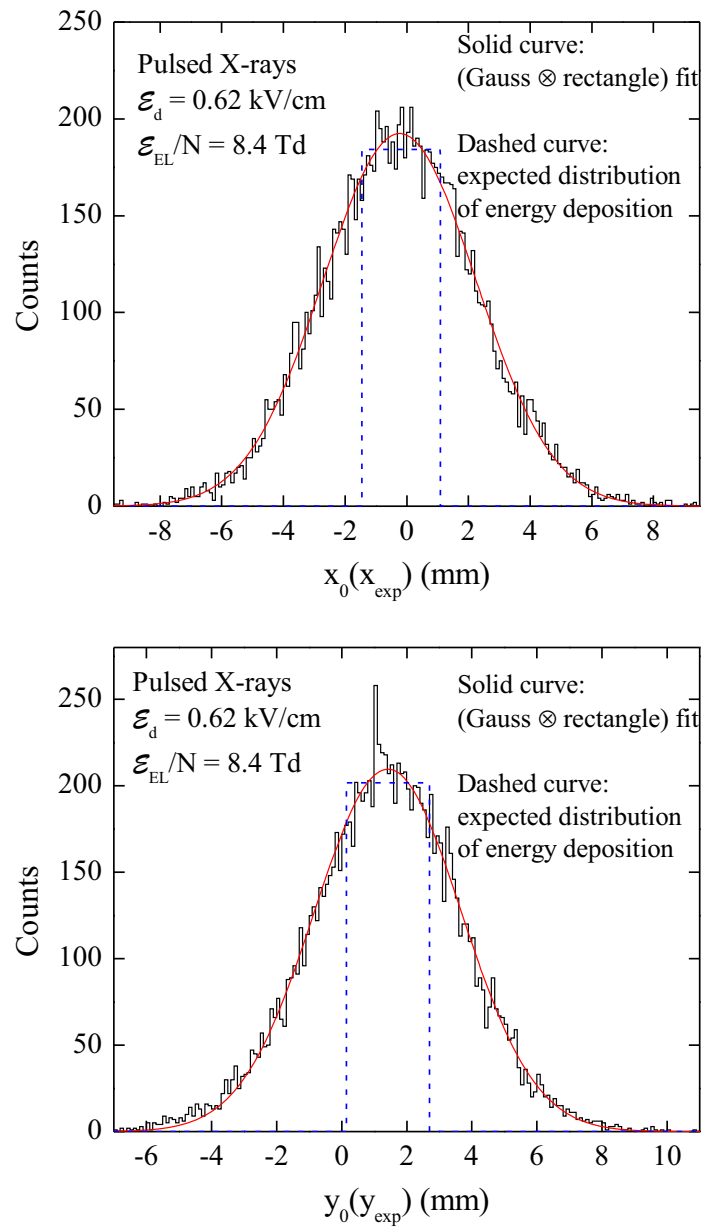

Fig. 20 Direct SiPM-matrix readout: projections of the 2D distribution presented in Fig. 19 on $x$ and $y$ axes. Also shown are the fit of the $x_{0}\left(x_{\text {exp }}\right)$ and $y_{0}\left(y_{\text {exp }}\right)$ distributions (red curves) and the expected distributions of the true coordinates of the interaction region (blue dotted curves), defined by the positions of the X-ray tube and collimator and by the X-ray range in liquid Ar. Note that here the signal is produced by several X-ray photons in a pulse, with the average energy of $25 \mathrm{keV}$, absorbed in a thin $(3 \mathrm{~mm})$ liquid Ar layer near the cathode

Figures 19, 20 and 21 characterize the detector performance at the maximum EL field. The position resolution and the average number of photoelectrons for lower fields were obtained in a similar way. These allow to define the dependence of the position resolution on the average total number of photoelectrons recorded by the SiPM matrix $\left(N_{P E}\right)$ : see Fig. 22.

The similar dependence, namely the position resolution as function of the total photoelectron number, was also obtained for the THGEM/SiPM-matrix readout. Here the ${ }^{109} \mathrm{Cd}$ source was used instead of pulsed X-rays, to avoid the problems related to electronics saturation induced by high photon flux in the latter case. The procedure to measure the position resolution with the ${ }^{109} \mathrm{Cd}$ source was generally similar to that with pulsed X-rays. The difference was that in the fit of $x_{0}\left(x_{\text {exp }}\right)$ and $y_{0}\left(y_{\text {exp }}\right)$ distributions the background due to Compton

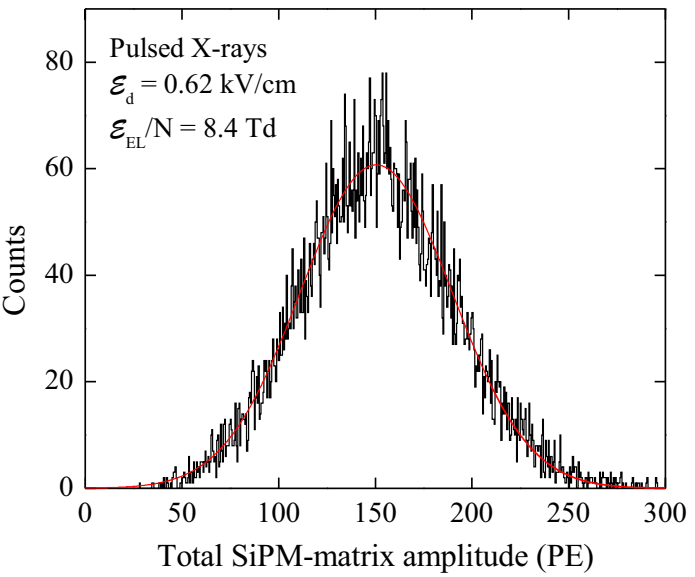

Fig. 21 Direct SiPM-matrix readout: amplitude spectrum of the total SiPM-matrix signal obtained with pulsed X-rays and $2 \mathrm{~mm}$ collimator. Red curve is fit by Gauss function. The data were obtained at the maximum reduced EL field, of $8.4 \mathrm{Td}$

scattering of gamma-rays was taken into account (described by a wide Gauss function). The position resolution was measured for different ${ }^{109} \mathrm{Cd}$ source energies, of $23.5 \mathrm{keV}$ and $82 \mathrm{keV}$, and for different THGEM charge gains, of 9 and 37. The resulting dependence is shown Fig. 22.

Looking at the figure one may conclude that the position resolution does not depend on the readout concept: it has a universal character, depending only on the total photolectron number recorded by the SiPM matrix $\left(N_{P E}\right)$, described by the inverse root function:

$\sigma=26 \mathrm{~mm} / \sqrt{N_{P E}}$.

This is surprising, since the readout geometry in both concepts is different. This universality might be due to the fact that in both readout concepts the THGEM1 electrode is used in front of the SiPM matrix, where THGEM1 holes act either as passive (light-transmitting) elements of an optical mask or as active (light-emitting) elements. Another possible explanation is that with a fairly large SiPM spacing in the SiPM matrix $(1 \mathrm{~cm})$, the difference in the distances to spatial regions where the light is produced for both readout concepts becomes insignificant.

\section{Discussion}

In this section we will try to estimate the detection thresholds in two-phase argon detectors with direct SiPM-matrix and THGEM/SiPM-matrix readout. The detection threshold is defined as the minimum energy, deposited by a scattered particle in the noble-gas liquid, that can be detected by the detector. There are two types of particle scattering: that of electron recoil, induced by gamma-ray and minimum ioniz- 


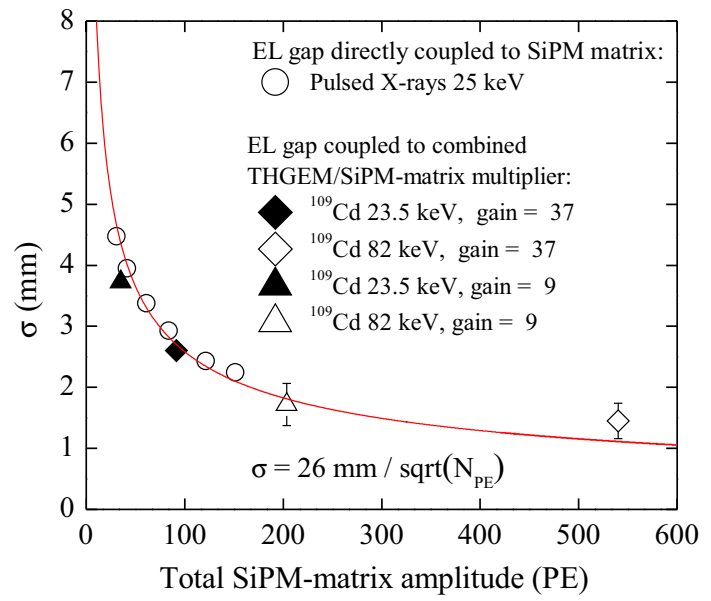

Fig. 22 Summary of position resolution results obtained in the twophase detector for the direct SiPM-matrix and THGEM/SiPM-matrix readout. Shown is the position resolution (standard deviation) as a function of the total number of photoelectrons recorded by the SiPM matrix. Red curve is the fit by inverse root function using all data points

ing particle scattering, or resulting from beta decays, such as by ${ }^{39} \mathrm{Ar}$, and that of nuclear recoil, induced by neutron and dark matter particle (WIMP) scattering. Their recoil energies are expressed in terms of keVee and keVnr respectively [1].

The EL gap yields obtained in this work, of $0.022 \mathrm{PE} / \mathrm{e}^{-}$ for direct SiPM-matrix readout and $0.65 \mathrm{PE} / \mathrm{e}^{-}$for THGEM/SiPM-matrix readout, can be significantly increased by optimizing the two-phase detector. Firstly, in direct SiPMmatrix readout, the THGEM1 anode with optical transparency (combined with the angle reduction factor) of only $0.28 \cdot 0.40=0.11$ can be replaced by the transparent electrode with ITO coating. Secondly, the sensitive area of the SiPM matrix can be increased from the current $36 \%$ (see Fig. 5c) to about $90 \%$, pushing the SiPMs close to each other. Consequently in optimized conditions, the amplitude yield can be increased up to about $0.5 \mathrm{PE} / \mathrm{e}^{-}$for direct SiPMmatrix readout (at EL reduced field of $8.4 \mathrm{Td}$ ) and up to 1.6 PE/e ${ }^{-}$for THGEM/SiPM-matrix readout (at THGEM1 charge gain of 37).

The detection threshold of an S2 signal depends on the pulse shape and dark count rate. For certainty, let the detection threshold (in terms of the photoelectron number) be $10 \mathrm{PE}$. Indeed, this value is large enough in terms of the position resolution (of about $1 \mathrm{~cm}$ ) and energy resolution (of about $30 \%$ assuming Poisson statistics). Now we can calculate the minimum number of electrons drifting in the EL gap, corresponding to $10 \mathrm{PE}$ signal at the SiPM matrix, using the amplitude yields of the previous paragraph. It amounts to $20 \mathrm{e}^{-}$and $6.2 \mathrm{e}^{-}$for direct SiPM-matrix and THGEM/SiPMmatrix readout respectively.

For a rough estimate of the energy thresholds, we used the ionization yield in liquid argon measured in [46] at low energies (around several $\mathrm{keVs}$ ): it is $10 \mathrm{e}^{-} / \mathrm{keVee}$ for electron recoils and $3.6 \mathrm{e}^{-} / \mathrm{keVnr}$ for nuclear recoils, at a drift field of $0.24 \mathrm{kV} / \mathrm{cm}$ (which is close to that used in DarkSide50 experiment [2]). Here we implied a full $100 \%$ electron transmission through THGEM0 electrode, since that can be easily reached by just increasing the voltage applied across THGEM0 [34]. The appropriate detection thresholds are presented in Table 2.

These values should be considered as just indicative. In particular the detection threshold for nuclear recoils for direct SiPM-matrix readout is of the order of $6 \mathrm{keVnr}$, which is enough to search WIMPs with masses above $10 \mathrm{GeV}$. For THGEM/SiPM-matrix readout the threshold is a factor of 3 lower, of the order of $2 \mathrm{keVnr}$, which is already close to that of DarkSide-50 experiment [4]. Moreover, it can be further decreased, by increasing the THGEM charge gain, for example by using the double-THGEM multiplier [7,26].

As an example, let us consider the detector response to a $1 \mathrm{MeV}$ signal induced by solar neutrino interaction, in particular when recording the CNO neutrinos as proposed in [3]. Taking into account the ionization yield from [47] and the EL gap yield from Table 2, for $1 \mathrm{MeV}$ signal the number of drifting electrons and photolectrons for the THGEM/SiPMmatrix readout would exceed 12,000 and 20,000 respectively, which would provide a sufficient energy resolution according to Poisson statistics, of about $1 \%$ root-mean-square.

Let us evaluate now the position resolution properties of the SiPM-matrix readout in comparison with a PMT-matrix readout. Table 3 compares the position resolution at a certain photoelectron number, reported in different dark matter search experiments using PMT-matrix readout, to that obtained in this work and extrapolated to the given photoelectron number using Eq. (3).

This extrapolation can only be considered indicative. Nevertheless it allows to conclude that the position resolution of the SiPM-matrix readout is always superior to that of PMTmatrix readout, by a factor varying from 3 to more than an order of magnitude. This superiority can be explained by a decrease in the channel pitch, from 3 in. in the case of PMTmatrix to $1 \mathrm{~cm}$ in the case of SiPM-matrix.

The obvious application of superior position resolution is the precise $\mathrm{x}-\mathrm{y}$ fiducialization, which can help reducing background from the TPC wall with minimal loss of fiducial mass. In addition, at sufficiently high event energy, high readout granularity can enable resolving two nearby scattering vertices with the same drift time.

In this work we considered the issue of S2-only signal detection, thus overlooking the problem of S1 signal detection. In this case, the background rejection should be similar to that applied in dark matter search experiments operated in S2-only detection mode $[4,5]$, in particular using effective passive TPC shielding and proper MC simulation. 
Table 2 Detection thresholds, corresponding to $10 \mathrm{PE}$ signal at the SiPM matrix, that can be achieved under optimal conditions for alternative readout concepts of the two-phase argon detectors with $1.8 \mathrm{~cm}$ thick EL gap, expressed in drifting electrons in the EL gap $\left(e^{-}\right)$and in

\begin{tabular}{|c|c|c|c|c|}
\hline Readout concept & \multicolumn{3}{|c|}{ Detection threshold for $10 \mathrm{PE}$} & $\begin{array}{l}\text { EL gap yield } \\
\left(\mathrm{PE} / \mathrm{e}^{-}\right)\end{array}$ \\
\hline Direct SiPM-matrix readout $\left(1.8 \mathrm{~cm}\right.$ thick EL gap, $\left.\mathcal{E}_{E L} / N=8.4 \mathrm{Td}\right)$ & 20 & 2 & 5.6 & 0.5 \\
\hline THGEM/SiPM-matrix readout $($ THGEM gain = 37) & 6.2 & 0.6 & 1.7 & 1.6 \\
\hline
\end{tabular}

Table 3 Position resolution of two-phase detectors in $x, y$ plane extrapolated from that obtained in this work (using SiPM-matrix readout with $1 \mathrm{~cm}$ channel pitch) in comparison with that reported in dark-matter search experiments (using PMT-matrix readout). Also shown is the data for dual-phase Xe TPC with SiPM-matrix readout [48]

\begin{tabular}{|c|c|c|c|}
\hline $\begin{array}{l}\text { able } 3 \text { Position resolution of } \\
\text { wo-phase detectors in } x, y \\
\text { lane extrapolated from that } \\
\text { btained in this work (using }\end{array}$ & Experiment & $\begin{array}{l}\text { Reported position } \\
\text { resolution }\end{array}$ & $\begin{array}{l}\text { Position resolution } \\
\text { expected for } \mathrm{SiPM} \\
\text { matrix with } 1 \mathrm{~cm} \\
\text { channel pitch }\end{array}$ \\
\hline $\begin{array}{l}\text { hannel pitch) in comparison } \\
\text { vith that reported in dark-matter }\end{array}$ & This work & $\sigma=\frac{26 \mathrm{~mm}}{\sqrt{N_{P E}}}$ & - \\
\hline earch experiments (using & LUX [49] & $\sigma=\frac{75 \mathrm{~mm}}{\sqrt{N_{P E}}}$ & $\sigma=\frac{26 \mathrm{~mm}}{\sqrt{N_{P E}}}$ \\
\hline $\begin{array}{l}\text { matrix readout). Also } \\
n \text { is the data for dual-phase }\end{array}$ & XENON100 [50] & $\sigma(46000 \mathrm{PE})=3 \mathrm{~mm}$ & $0.12 \mathrm{~mm}$ \\
\hline TPC with SiPM-matrix & XENON1T [51] & $\sigma(200 \mathrm{PE})=20 \mathrm{~mm}$ & $1.8 \mathrm{~mm}$ \\
\hline out [48] & DarkSide-50 [52] & $\sigma(20000 \mathrm{PE})=6 \mathrm{~mm}$ & $0.18 \mathrm{~mm}$ \\
\hline & Dual-phase Xe TPC with SiPM matrix [48] & $\sigma(1000 \mathrm{PE})=1.5 \mathrm{~mm}$ & $0.82 \mathrm{~mm}$ \\
\hline
\end{tabular}

deposited energy in liquid Ar for electron (keVee) and nuclear (keVnr) recoils, at a drift field in liquid Ar of $0.24 \mathrm{kV} / \mathrm{cm}$. Also shown are the EL gap yields
Finally, one should mention the possibility to record the S1 signal directly, without TPB, i.e. similarly to that of S2 in this work: using the effect of primary scintillations in the visible range in liquid Ar observed elsewhere [14,53], albeit at lower light yield compared to ordinary (VUV) scintillation.

\section{Conclusions}

In this work, we have for the first time demonstrated two alternative techniques of the SiPM-matrix readout of two-phase argon detectors, using electroluminescence (EL) in the visible and NIR range induced by either neutral bremsstrahlung $(\mathrm{NBrS})$ or avalanche scintillation.

In the first technique, the EL gap was directly read out by the SiPM matrix. In the second technique, the EL gap was read out via combined THGEM/SiPM-matrix multiplier, the THGEM being operated in electron avalanche mode.

The amplitude yield was measured for these readout techniques: under optimal conditions it would amount to about $0.5 \mathrm{PE} / \mathrm{e}^{-}$and $1.6 \mathrm{PE} / \mathrm{e}^{-}$for the direct SiPM-matrix and THGEM/SiPM-matrix readout respectively. This allowed to assess the detection threshold in two-phase argon detectors for dark matter search: for nuclear recoils it was estimated to be of the order of $6 \mathrm{keVnr}$ and $2 \mathrm{keVnr}$, respectively.

Using the SiPM matrix with $1 \mathrm{~cm}$ channel pitch, we obtained the highest position resolution ever measured for two-phase detectors with an EL gap: $\sigma=26 \mathrm{~mm} / \sqrt{N_{P E}}$.

Unlike the "standard" optical readout of two-phase TPCs (in the VUV), both alternative readout techniques allow to operate without TPB, which is particularly valuable for largescale detectors. In particular, the results of this study were intended for use in the DarkSide-20k experiment: the alternative readout techniques might be considered as backup solutions, in case issues with TPB instability over time or non uniformity over large areas should become problematic.

There is another possible application of the NBrS EL signal in the DarkSide experiment. Due to its fast nature, its pulse width can be used to accurately measure the EL gap thickness even under current experimental conditions (i.e. using WLS), provided that the EL gap operates at lower fields (below $4 \mathrm{Td}$ ), where the S2 slow component of ordinary EL disappears and thus does not interfere with measurements.

Finally, it should be emphasized that, to the best of our knowledge, this is the first practical application of the $\mathrm{NBrS}$ effect in detection science.

Acknowledgements The minor part of the work, regarding the study of SiPM matrices in sections 3, was supported by Russian Foundation for Basic Research (project no. 18-02-00117). The major part of the work, including the results on EL yield and SiPM-matrix readout in sections 4-10, was supported by Russian Science Foundation (project no. 20-12-00008), The work was done within the $R \& D$ program for the DarkSide-20k experiment.

Data Availability Statement This manuscript has no associated data or the data will not be deposited. [Authors' comment: The data will be available at any time by contacting the Corresponding Author.]

Open Access This article is licensed under a Creative Commons Attribution 4.0 International License, which permits use, sharing, adaptation, distribution and reproduction in any medium or format, as long as you give appropriate credit to the original author(s) and the source, pro- 
vide a link to the Creative Commons licence, and indicate if changes were made. The images or other third party material in this article are included in the article's Creative Commons licence, unless indicated otherwise in a credit line to the material. If material is not included in the article's Creative Commons licence and your intended use is not permitted by statutory regulation or exceeds the permitted use, you will need to obtain permission directly from the copyright holder. To view a copy of this licence, visit http://creativecomm ons.org/licenses/by/4.0/.

Funded by SCOAP ${ }^{3}$.

\section{References}

1. V. Chepel, H. Araujo, J. Instrum. 8, R04001 (2013). https://doi. org/10.1088/1748-0221/8/04/R04001

2. P. Agnes et al., Phys. Lett. B 743, 456 (2015). https://doi.org/10. 1016/j.physletb.2015.03.012

3. C.E. Aalseth et al., Eur. Phys. J. Plus 133, 131 (2018). https://doi. org/10.1140/epjp/i2018-11973-4

4. P. Agnes et al., Phys. Rev. Lett. 121, 081307 (2018). https://doi. org/10.1103/PhysRevLett.121.081307

5. E. Aprile et al., Phys. Rev. Lett. 123, 251801 (2019). https://doi. org/10.1103/PhysRevLett.123.251801

6. C. Oliveira et al., Phys. Lett. B 703, 217 (2011). https://doi.org/10. 1016/j.physletb.2011.07.081

7. A. Buzulutskov, Instrumentation 4, 16 (2020). https://doi.org/10. 3390/instruments4020016

8. C.E. Aalseth et al., J. Instrum. 12, P09030 (2017). https://doi.org/ 10.1088/1748-0221/12/09/p09030

9. F. Acerbi et al., IEEE Trans. Electron Devices 64, 521 (2017). https://doi.org/10.1109/TED.2016.2641586

10. T. Igarashi et al., Nucl. Instrum. Methods Phys. Res. A 833, 239 (2016). https://doi.org/10.1016/j.nima.2016.07.008

11. A. Ghassemi (Hamamatsu Photonics), XIV International Conference on Topics in Astroparticle and Underground Physics 7-11 September 2015 "Torino" Italy

12. J. Asaadi et al., J. Instrum. 14, P02021 (2019). https://doi.org/10. 1088/1748-0221/14/02/P02021

13. B. Burak et al., J. Instrum. 15, C04021 (2020). https://doi.org/10. 1088/1748-0221/15/04/C04021

14. A. Buzulutskov et al., Europhys. Lett. 94, 52001 (2011). https:// doi.org/10.1209/0295-5075/94/52001

15. A. Buzulutskov et al., Astropart. Phys. 103, 29 (2018). https://doi. org/10.1016/j.astropartphys.2018.06.005

16. A. Bondar et al., Nucl. Instrum. Methods Phys. Res. A 958, 162432 (2020). https://doi.org/10.1016/j.nima.2019.162432

17. P. Lindblom, O. Solin, Nucl. Instrum. Methods Phys. Res. A 268, 204 (1988). https://doi.org/10.1016/0168-9002(88)90607-9

18. M.M. Fraga et al., IEEE Trans. Nucl. Sci. 47, 933 (2000). https:// doi.org/10.1109/23.856721

19. A. Bondar et al., J. Instrum. 7, P06014 (2012). https://doi.org/10. 1088/1748-0221/7/06/P06014

20. C. Oliveira et al., Nucl. Instrum. Methods Phys. Res. A 722, 1 (2013). https://doi.org/10.1016/j.nima.2013.04.061

21. A. Bondar et al., J. Instrum. 5, P08002 (2010). https://doi.org/10. 1088/1748-0221/5/08/P08002

22. A. Morozov et al., J. Appl. Phys. 103, 103301 (2008). https://doi. org/10.1063/1.2931000

23. http://www.hamamatsu.com
24. A. Otte et al., Nucl. Instrum. Methods Phys. Res. A 846, 106 (2017). https://doi.org/10.1016/j.nima.2016.09.053

25. C. Monteiro et al., Phys. Lett. B 668, 167 (2008). https://doi.org/ 10.1016/j.physletb.2008.08.030

26. A. Buzulutskov, J. Instrum. 7, C02025 (2012). https://doi.org/10. 1088/1748-0221/7/02/C02025

27. A. Bondar et al., Nucl. Instrum. Methods Phys. Res. A 732, 213 (2013). https://doi.org/10.1016/j.nima.2013.07.089

28. A. Bondar et al., J. Instrum. 3, P07001 (2008). https://doi.org/10. 1088/1748-0221/3/07/P07001

29. http://www.cpta-apd.ru; http://www.photonique.ch

30. A. Bondar et al., Nucl. Instrum. Methods Phys. Res. A 628, 364 (2011). https://doi.org/10.1016/j.nima.2010.07.002

31. A. Bondar et al., J. Instrum. 10, P04013 (2015). https://doi.org/10. 1088/1748-0221/10/04/P04013

32. A. Bondar et al., Nucl. Instrum. Methods Phys. Res. A 845, 206 (2017). https://doi.org/10.1016/j.nima.2016.05.109

33. A. Breskin et al., Nucl. Instrum. Methods Phys. Res. A 598, 107 (2009). https://doi.org/10.1016/j.nima.2008.08.062

34. A. Bondar et al., Nucl. Instrum. Methods Phys. Res. A 943, 162431 (2019). https://doi.org/10.1016/j.nima.2019.162431

35. A. Bondar et al., J. Instrum. 15, C06053 (2020). https://doi.org/10. 1088/1748-0221/15/06/C06053

36. A. Bondar et al., J. Instrum. 10, P10010 (2015). https://doi.org/10. 1088/1748-0221/10/10/P10010

37. V. Gehman et al., J. Instrum. 8, P04024 (2013). https://doi.org/10. 1088/1748-0221/8/04/P04024

38. A. Bondar et al., Nucl. Instrum. Methods Phys. Res. A 816, 119 (2016). https://doi.org/10.1016/j.nima.2016.02.010

39. A. Bondar et al., Instrum. Exp. Tech. 62, 746 (2019). https://doi. org/10.1134/S0020441219050142

40. A. Bondar et al., J. Instrum. 12, C05016 (2017). https://doi.org/10. 1088/1748-0221/12/05/C05016

41. A. Bondar et al., J. Instrum. 8, P02008 (2013). https://doi.org/10. 1088/1748-0221/8/02/P02008

42. G. Landi, Nucl. Instrum. Methods Phys. Res. A 485, 698 (2002). https://doi.org/10.1016/S0168-9002(01)02071-X

43. A. Fabbri et al., J. Instrum. 8, P12010 (2013). https://doi.org/10. 1088/1748-0221/8/12/P12010

44. A. Bondar et al., EPJ Web Conf. 174 (2018). https://doi.org/10. 1051/epjconf/201817402005

45. V.N. Solovov et al., IEEE Trans. Nucl. Sci. 59, 3286 (2012). https:// doi.org/10.1109/TNS.2012.2221742

46. T.H. Joshi et al., Phys. Rev. Lett. 112, 171303 (2014). https://doi. org/10.1103/PhysRevLett.112.171303

47. E. Shibamura et al., Nucl. Instrum. Methods 131, 249 (1975). https://doi.org/10.1016/0029-554X(75)90327-4

48. L. Baudis et al., Eur. Phys. J. C 80, 477 (2020). https://doi.org/10. 1140/epjc/s10052-020-8031-6

49. D.S. Akerib et al., J. Instrum. 13, P02001 (2018). https://doi.org/ 10.1088/1748-0221/13/02/P02001

50. E. Aprile et al., Astropart. Phys. 35, 573 (2012). https://doi.org/10. 1016/j.astropartphys.2012.01.003

51. E. Aprile et al., Phys. Rev. Lett. 119, 181301 (2017). https://doi. org/10.1103/PhysRevLett.119.181301

52. P. Agnes et al., Phys. Rev. D 98, 102006 (2018). https://doi.org/10. 1103/PhysRevD.98.102006

53. T. Alexander et al., J. Instrum. 11, C03010 (2016). https://doi.org/ $10.1088 / 1748-0221 / 11 / 03 / \mathrm{C} 03010$ 


\section{The DarkSide-20k collaboration}

C. E. Aalseth ${ }^{1}$, S. Abdelhakim ${ }^{2}$, P. Agnes ${ }^{3}$, R. Ajaj ${ }^{4}$, I. F. M. Albuquerque ${ }^{5}$, T. Alexander ${ }^{1}$, A. Alici ${ }^{6,7}$, A. K. Alton ${ }^{8}$, P. Amaudruz ${ }^{9}$, F. Ameli ${ }^{10}$, J. Anstey ${ }^{4}$, P. Antonioli ${ }^{7}$, M. Arba ${ }^{11}$, S. Arcelli ${ }^{6,7}$, R. Ardito ${ }^{12,13}$, I. J. Arnquist ${ }^{1}$, P. Arpaia ${ }^{14,15}$, D. M. Asner ${ }^{16}$, A. Asunskis ${ }^{17}$, M. Ave ${ }^{5}$, H. O. Back ${ }^{1}$, V. Barbaryan ${ }^{18}$, A. Barrado Olmedo ${ }^{19}$, G. Batignani ${ }^{20,21}$, M. G. Bisogni ${ }^{20,21}$, V. Bocci ${ }^{10}$, A. Bondar ${ }^{22,23}$, G. Bonfini ${ }^{24}$, W. Bonivento ${ }^{11}$, E. Borisova ${ }^{22,23}$, B. Bottino ${ }^{25,26}$, M. G. Boulay ${ }^{4}$, R. Bunker ${ }^{1}$, S. Bussino ${ }^{27,28}$, A. Buzulutskov ${ }^{22,23}$, M. Cadeddu ${ }^{11,29}$, M. Cadoni ${ }^{11,29}$, A. Caminata ${ }^{26}$, N. Canci ${ }^{3,24}$, A. Candela ${ }^{24}$, C. Cantini ${ }^{30}$, M. Caravati ${ }^{11}, \quad$ M. Cariello ${ }^{26}$, F. Carnesecchi ${ }^{6,7,31}$, A. Castellani ${ }^{12,13}$, P. Castello ${ }^{11,32}$, P. Cavalcante ${ }^{24,33}$, D. Cavazza ${ }^{7}, \quad$ S. Cavuoti ${ }^{15,34}$, S. Cebrian ${ }^{35}$, J. M. Cela Ruiz ${ }^{19}$, B. Celano ${ }^{15}$, R. Cereseto ${ }^{26}$, S. Chashin ${ }^{18}$, W. Cheng ${ }^{36,37}$, A. Chepurnov ${ }^{18}$, C. Cicalò ${ }^{11}$, L. Cifarelli ${ }^{6,7}$, M. Citterio ${ }^{13}$, F. Coccetti ${ }^{31}$, V. Cocco $^{11}$, M. Colocci ${ }^{6,7}$, E. Conde Vilda ${ }^{19}$, L. Consiglio ${ }^{38}$, F. Cossio ${ }^{36,37}$, G. Covone ${ }^{15,34}$, P. Crivelli ${ }^{30}$, I. D'Antone ${ }^{7}$, M. D’Incecco ${ }^{24}$, M. D. Da Rocha Rolo ${ }^{36}$ ，O. Dadoun ${ }^{39}$ ， M. Daniel ${ }^{19}$ ， S. Davini ${ }^{26}$ ， S. De Cecco ${ }^{10,40}$ ， M. De Deo ${ }^{24}$, A. De Falco ${ }^{11,29}$, D. De Gruttola ${ }^{41,42}$, G. De Guido ${ }^{13,43}$ ， G. De Rosa ${ }^{15,34}$, G. Dellacasa ${ }^{36}$, P. Demontis ${ }^{44,45,46}$, S. De Pasquale ${ }^{41,42}$, A. V. Derbin ${ }^{47}$, A. Devoto ${ }^{11,29}$, F. Di Eusanio ${ }^{24,48}$, L. Di Noto ${ }^{25,26}$, G. Di Pietro ${ }^{13,24}$, P. Di Stefano ${ }^{49}$, C. Dionisi ${ }^{10,40}$, G. Dolganov ${ }^{50}$, F. Dordei ${ }^{11}$, M. Downing ${ }^{51}$, F. Edalatfar ${ }^{9}$, A. Empl ${ }^{3}$, M. Fernandez Diaz ${ }^{19}$, C. Filip ${ }^{52}$, G. Fiorillo ${ }^{15,34}$, K. Fomenko ${ }^{53}$, A. Franceschi ${ }^{54}$, D. Franco ${ }^{55}$, E. Frolov ${ }^{22,23}$, G. E. Froudakis ${ }^{56}$, N. Funicello ${ }^{41,42}$, F. Gabriele ${ }^{24}$, A. Gabrieli ${ }^{44,45}$, C. Galbiati ${ }^{38,48}$, M. Garbini ${ }^{7,31}$, P. Garcia Abia ${ }^{19}$, D. Gascón Fora ${ }^{57}$, A. Gendotti ${ }^{30}$, C. Ghiano ${ }^{24}$, A. Ghisi ${ }^{12,13}$, P. Giampa ${ }^{9}$, R. A. Giampaolo ${ }^{36,37}$, C. Giganti ${ }^{39}$, M. A. Giorgi ${ }^{20,21}$, G. K. Giovanetti ${ }^{48}$, $\begin{array}{lll}\text { M. L. Gligan } & \text { O. Gorchakov } \\ & \end{array}$ M. Gromov ${ }^{18,53}$, M. Guan ${ }^{60}$, M. B. B. Guerra ${ }^{17}$, M. Guerzoni ${ }^{7}$, M. Gulino ${ }^{45,61}$, R. K. Haaland ${ }^{62}$, B. R. Hackett ${ }^{1}$, A. Hallin ${ }^{63}$, M. Haranczyk ${ }^{58}$, B. Harrop ${ }^{48}$, E. W. Hoppe ${ }^{1}$, S. Horikawa ${ }^{24,38}$, B. Hosseini ${ }^{11}$, F. Hubaut ${ }^{64}$, P. Humble ${ }^{1}$, E. V. Hungerford ${ }^{3}$, An. Ianni ${ }^{44,48}$, A. Ilyasov ${ }^{50,59}$, V. Ippolito ${ }^{10}$, C. Jillings ${ }^{65,66}$, K. Keeter ${ }^{17}$, C. L. Kendziora ${ }^{67}$, I. Kochanek ${ }^{24}$, K. Kondo ${ }^{38}$, G. Kopp ${ }^{48}$, D. Korablev ${ }^{53}$, G. Korga ${ }^{3,24}$, A. Kubankin ${ }^{68}$, R. Kugathasan ${ }^{36,37}$, M. Kuss ${ }^{20}$, M. La Commara ${ }^{15,69}$, L. La Delfa ${ }^{11}$, M. Lai1 ${ }^{1,29}$, M. Lebois ${ }^{2}$, B. Lehnert ${ }^{63}$, N. Levashko ${ }^{50,59}$, X. Li ${ }^{48}$, Q. Liqiang ${ }^{2}$, M. Lissia ${ }^{11}$, G. U. Lodi $^{13,43}$, G. Longo ${ }^{15,34}$, R. Lussana ${ }^{13,70}$, L. Luzzi ${ }^{13,71}$, A. A. Machado ${ }^{72}$, I. N. Machulin ${ }^{50,59}$, A. Mandarano ${ }^{24,38}$, S. Manecki ${ }^{49,66}$, L. Mapelli ${ }^{48}$, A. Margotti ${ }^{7}$, S. M. Mari ${ }^{27,28}$, M. Mariani13,71, J. Maricic ${ }^{73}$, M. Marinelli ${ }^{25,26}$, D. Marras ${ }^{11}$, M. Martínez ${ }^{35,74}$, A. D. Martinez Rojas ${ }^{36,37}$, M. Mascia ${ }^{11,75}$, J. Mason ${ }^{4}$, A. Masoni ${ }^{11}$, A. B. McDonald ${ }^{49}$, A. Messina ${ }^{10,40}$, T. Miletic ${ }^{73}$, R. Milincic ${ }^{73}$, A. Moggi ${ }^{20}$, S. Moioli ${ }^{13,43}$, J. Monroe ${ }^{76}$, M. Morrocchi ${ }^{20}$,

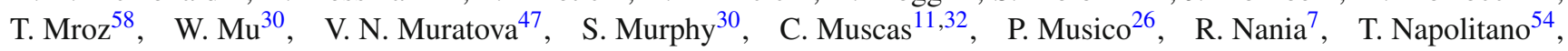
A. Navrer Agasson ${ }^{39}$, M. Nessi ${ }^{77}$, I. Nikulin ${ }^{68}$, V. Nosov ${ }^{22,23}$, J. A. Nowak ${ }^{78}$, A. Oleinik ${ }^{68}$, V. Oleynikov ${ }^{22,23}$, M. Orsini ${ }^{24}$, F. Ortica ${ }^{79,80}$, L. Pagani ${ }^{81}$, M. Pallavicini ${ }^{25,26}$, S. Palmas ${ }^{11,75}$, L. Pandola ${ }^{45}$, E. Pantic ${ }^{81}$, E. Paoloni ${ }^{20,21}$, F. Pazzona ${ }^{44,45}$, S. Peeters ${ }^{82}$, P. A. Pegoraro ${ }^{11,32}$, K. Pelczar ${ }^{58}$, L. A. Pellegrini ${ }^{13,43}$, C. Pellegrino ${ }^{7,31}$, N. Pelliccia ${ }^{79,80}$, F. Perotti ${ }^{12,13}$, V. Pesudo ${ }^{19}$, E. Picciau ${ }^{11,29}$, F. Pietropaolo ${ }^{77}$, A. Pocar ${ }^{51}$, T. R. Pollmann ${ }^{83}$, D. Portaluppi ${ }^{13,70}$, S. S. Poudel $^{3}$, P. Pralavorio ${ }^{64}$, D. Price ${ }^{84}$, B. Radics ${ }^{30}$, F. Raffaelli20, F. Ragusa ${ }^{13,85}$, M. Razeti ${ }^{11}$, C. Regenfus ${ }^{30}$, A. L. Renshaw ${ }^{3}$, S. Rescia ${ }^{16}$, M. Rescigno ${ }^{10}$, F. Retiere ${ }^{9}$, L. P. Rignanese ${ }^{6,7,31}$, C. Ripoli ${ }^{41,42}$, A. Rivetti ${ }^{36}$, J. Rode ${ }^{39,55}$, A. Romani ${ }^{79,80}$, L. Romero ${ }^{19}$, N. Rossi ${ }^{10,24}$, A. Rubbia ${ }^{30}$, P. Sala ${ }^{77}$, P. Salatino ${ }^{15,86}$, O. Samoylov ${ }^{53}$, E. Sánchez García ${ }^{19}$, E. Sandford ${ }^{84}$, S. Sanfilippo ${ }^{27,28}$, M. Sant ${ }^{44,45}$, D. Santone ${ }^{76}$, R. Santorelli ${ }^{19}$, C. Savarese ${ }^{48}$, E. Scapparone ${ }^{7}$, B. Schlitzer ${ }^{81}$, G. Scioli ${ }^{6,7}$, E. Segreto ${ }^{72}$, A. Seifert ${ }^{1}$, D. A. Semenov ${ }^{47}$, A. Shchagin ${ }^{68}$, A. Sheshukov ${ }^{53}$, S. Siddhanta ${ }^{11}$, M. Simeone ${ }^{15,86}$, P. N. Singh ${ }^{3}$, P. Skensved ${ }^{49}$, M. D. Skorokhvatov ${ }^{50,59}$, O. Smirnov ${ }^{53}$, G. Sobrero ${ }^{26}$, A. Sokolov ${ }^{22,23}$, A. Sotnikov ${ }^{53}$, R. Stainforth ${ }^{4}$, A. Steri ${ }^{11}$, S. Stracka ${ }^{20}$, V. Strickland ${ }^{4}$, G. B. Suffritti ${ }^{44,45,46}$, S. Sulis ${ }^{11,32}$, Y. Suvorov ${ }^{15,34,50, \text { A. M. Szelc }}{ }^{84}$, R. Tartaglia ${ }^{24}$, G. Testera ${ }^{26}$, T. Thorpe ${ }^{24,38}$, A. Tonazzo ${ }^{55}$, A. Tosi ${ }^{13,70}$, M. Tuveri ${ }^{11}$, E. V. Unzhakov ${ }^{47}$, G. Usai ${ }^{11,29}$, A. Vacca ${ }^{11,75}$, E. Vázquez-Jáuregui ${ }^{87}$, T. Viant ${ }^{30}$, S. Viel ${ }^{4}$, F. Villa ${ }^{13,70}$, A. Vishneva ${ }^{53}$, R. B. Vogelaar ${ }^{33}$, J. Wahl ${ }^{1}$, J. J. Walding ${ }^{76}$, H. Wang ${ }^{88}$, Y. Wang ${ }^{88}$, S. Westerdale ${ }^{4}$, R. J. Wheadon ${ }^{36}$, R. Williams ${ }^{1}$, J. Wilson ${ }^{2}$, Ma. M. Wojcik ${ }^{58}$, Ma. Wojcik ${ }^{89}$,

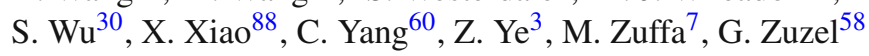

\footnotetext{
${ }^{1}$ Pacific Northwest National Laboratory, Richland, WA 99352, USA

${ }^{2}$ Institut de Physique Nuclèaire d'Orsay, 91406 Orsay, France

${ }^{3}$ Department of Physics, University of Houston, Houston, TX 77204, USA

${ }^{4}$ Department of Physics, Carleton University, Ottawa, ON K1S 5B6, Canada

${ }^{5}$ Instituto de Física, Universidade de São Paulo, São Paulo 05508-090, Brazil

${ }^{6}$ Physics Department, Università degli Studi di Bologna, 40126 Bologna, Italy

${ }^{7}$ INFN Bologna, 40126 Bologna, Italy

${ }^{8}$ Physics Department, Augustana University, Sioux Falls, SD 57197, USA
} 
${ }^{9}$ TRIUMF, 4004 Wesbrook Mall, Vancouver, BC V6T 2A3, Canada

${ }^{10}$ INFN Sezione di Roma, 00185 Rome, Italy

${ }^{11}$ INFN Cagliari, 09042 Cagliari, Italy

12 Civil and Environmental Engineering Department, Politecnico di Milano, 20133 Milan, Italy

${ }^{13}$ INFN Milano, 20133 Milan, Italy

${ }^{14}$ Department of Electrical Engineering and Information Technology, Università degli Studi "Federico II" di Napoli, 80125 Naples, Italy

${ }^{15}$ INFN Napoli, 80126 Naples, Italy

16 Brookhaven National Laboratory, Upton, NY 11973, USA

${ }^{17}$ School of Natural Sciences, Black Hills State University, Spearfish, SD 57799, USA

${ }^{18}$ Skobeltsyn Institute of Nuclear Physics, Lomonosov Moscow State University, Moscow 119234, Russia

${ }^{19}$ CIEMAT, Centro de Investigaciones Energéticas, Medioambientales y Tecnológicas, 28040 Madrid, Spain

${ }^{20}$ INFN Pisa, 56127 Pisa, Italy

${ }^{21}$ Physics Department, Università degli Studi di Pisa, 56127 Pisa, Italy

${ }^{22}$ Budker Institute of Nuclear Physics, Novosibirsk 630090, Russia

${ }^{23}$ Novosibirsk State University, Novosibirsk 630090, Russia

${ }^{24}$ INFN Laboratori Nazionali del Gran Sasso, 67100 Assergi, AQ, Italy

${ }^{25}$ Physics Department, Università degli Studi di Genova, 16146 Genoa, Italy

${ }^{26}$ INFN Genova, 16146 Genoa, Italy

${ }^{27}$ INFN Roma Tre, 00146 Rome, Italy

${ }^{28}$ Mathematics and Physics Department, Università degli Studi Roma Tre, 00146 Rome, Italy

${ }^{29}$ Physics Department, Università degli Studi di Cagliari, 09042 Cagliari, Italy

${ }^{30}$ Institute for Particle Physics, ETH Zürich, 8093 Zurich, Switzerland

${ }^{31}$ Museo della fisica e Centro studi e Ricerche Enrico Fermi, 00184 Rome, Italy

${ }^{32}$ Department of Electrical and Electronic Engineering, Università degli Studi, 09023 Cagliari, Italy

${ }^{33}$ Virginia Tech, Blacksburg, VA 24061, USA

${ }^{34}$ Physics Department, Università degli Studi “Federico II" di Napoli, 80126 Naples, Italy

${ }^{35}$ Centro de Astropartículas y Física de Altas Energías, Universidad de Zaragoza, 50009 Zaragoza, Spain

${ }^{36}$ INFN Torino, 10125 Turin, Italy

${ }^{37}$ Department of Electronics and Communications, Politecnico di Torino, 10129 Turin, Italy

${ }^{38}$ Gran Sasso Science Institute, 67100 L'Aquila, Italy

${ }^{39}$ LPNHE, CNRS/IN2P3, Sorbonne Université, Université Paris Diderot, 75252 Paris, France

${ }^{40}$ Physics Department, Sapienza Università di Roma, 00185 Rome, Italy

${ }^{41}$ Physics Department, Universitá degli Studi di Salerno, 84084 Salerno, Italy

${ }^{42}$ INFN Salerno, 84084 Salerno, Italy

${ }^{43}$ Chemistry, Materials and Chemical Engineering Department "G. Natta”, Politecnico di Milano, 20133 Milan, Italy

${ }^{44}$ Chemistry and Pharmacy Department, Università degli Studi di Sassari, 07100 Sassari, Italy

45 INFN Laboratori Nazionali del Sud, 95123 Catania, Italy

${ }^{46}$ Interuniversity Consortium for Science and Technology of Materials, 50121 Florence, Italy

${ }^{47}$ Saint Petersburg Nuclear Physics Institute, Gatchina 188350, Russia

${ }^{48}$ Physics Department, Princeton University, Princeton, NJ 08544, USA

${ }^{49}$ Department of Physics, Engineering Physics and Astronomy, Queen's University, Kingston, ON K7L 3N6, Canada

${ }^{50}$ National Research Centre Kurchatov Institute, Moscow 123182, Russia

${ }^{51}$ Amherst Center for Fundamental Interactions and Physics Department, University of Massachusetts, Amherst, MA 01003, USA

52 National Institute for R\&D of Isotopic and Molecular Technologies, 400293 Cluj-Napoca, Romania

53 Joint Institute for Nuclear Research, Dubna 141980, Russia

${ }^{54}$ INFN Laboratori Nazionali di Frascati, 00044 Frascati, Italy

55 APC, Université Paris Diderot, CNRS/IN2P3, CEA/Irfu, Obs de Paris, USPC, 75205 Paris, France

${ }^{56}$ Department of Chemistry, University of Crete, P.O. Box 2208, 71003 Heraklion, Crete, Greece

${ }^{57}$ Universitat de Barcelona, 08028 Barcelona, Catalonia, Spain

${ }^{58}$ M. Smoluchowski Institute of Physics, Jagiellonian University, 30-348 Kraków, Poland

${ }^{59}$ National Research Nuclear University MEPhI, Moscow 115409, Russia 
${ }^{60}$ Institute of High Energy Physics, Beijing 100049, China

${ }^{61}$ Engineering and Architecture Faculty, Università di Enna Kore, 94100 Enna, Italy

${ }^{62}$ Department of Physics and Engineering, Fort Lewis College, Durango, CO 81301, USA

${ }^{63}$ Department of Physics, University of Alberta, Edmonton, AB T6G 2R3, Canada

${ }^{64}$ Centre de Physique des Particules de Marseille, Aix Marseille Univ, CNRS/IN2P3, CPPM, Marseille, France

${ }^{65}$ Department of Physics and Astronomy, Laurentian University, Sudbury, ON P3E 2C6, Canada

${ }^{66}$ SNOLAB, Lively, ON P3Y 1N2, Canada

${ }^{67}$ Fermi National Accelerator Laboratory, Batavia, IL 60510, USA

${ }^{68}$ Radiation Physics Laboratory, Belgorod National Research University, Belgorod 308007, Russia

${ }^{69}$ Pharmacy Department, Università degli Studi “Federico II” di Napoli, 80131 Naples, Italy

${ }^{70}$ Electronics, Information, and Bioengineering Department, Politecnico di Milano, 20133 Milan, Italy

${ }^{71}$ Energy Department, Politecnico di Milano, 20133 Milan, Italy

72 Physics Institute, Universidade Estadual de Campinas, Campinas 13083, Brazil

${ }^{73}$ Department of Physics and Astronomy, University of Hawai'i, Honolulu, HI 96822, USA

${ }^{74}$ ARAID, Fundación Agencia Aragonesa para la Investigación y el Desarrollo, Gobierno de Aragón, 50018 Zaragoza, Spain

${ }^{75}$ Department of Mechanical, Chemical, and Materials Engineering, Università degli Studi, 09042 Cagliari, Italy

${ }^{76}$ Department of Physics, Royal Holloway University of London, Egham TW20 0EX, UK

${ }^{77}$ CERN, European Organization for Nuclear Research, 1211 Geneva 23, Switzerland

${ }^{78}$ Lancaster University, Lancaster LA1 4YW, UK

${ }^{79}$ Chemistry, Biology and Biotechnology Department, Università degli Studi di Perugia, 06123 Perugia, Italy

${ }^{80}$ INFN Perugia, 06123 Perugia, Italy

${ }^{81}$ Department of Physics, University of California, Davis, CA 95616, USA

82 Physics and Astronomy, University of Sussex, Brighton BN1 9QH, UK

${ }^{83}$ Physik Department, Technische Universität München, 80333 Munich, Germany

84 The University of Manchester, Manchester M13 9PL, UK

${ }^{85}$ Physics Department, Università degli Studi di Milano, 20133 Milan, Italy

${ }^{86}$ Chemical, Materials, and Industrial Production Engineering Department, Università degli Studi "Federico II" di Napoli, 80126 Naples, Italy

${ }^{87}$ Instituto de Física, Universidad Nacional Autónoma de México (UNAM), 01000 México, Mexico

${ }^{88}$ Physics and Astronomy Department, University of California, Los Angeles, CA 90095, USA

${ }^{89}$ Institute of Applied Radiation Chemistry, Lodz University of Technology, 93-590 Lodz, Poland 